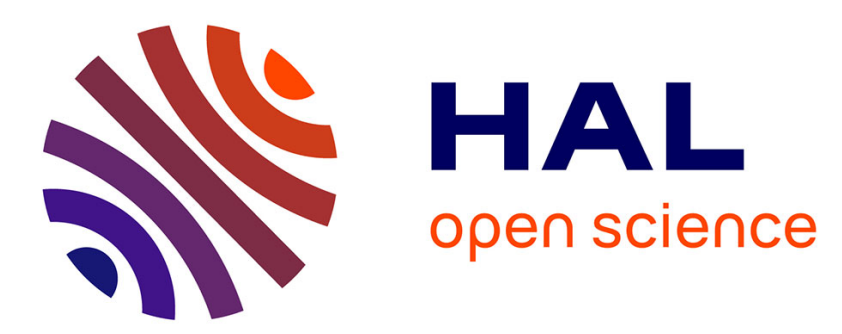

\title{
Integral representation and relaxation of local functionals on Cheeger-Sobolev spaces
}

Omar Anza Hafsa, Jean-Philippe Mandallena

\section{To cite this version:}

Omar Anza Hafsa, Jean-Philippe Mandallena. Integral representation and relaxation of local functionals on Cheeger-Sobolev spaces. Nonlinear Analysis: Theory, Methods and Applications, 2022, 217, 10.1016/j.na.2021.112744 . hal-03270938v2

\section{HAL Id: hal-03270938 \\ https://hal.science/hal-03270938v2}

Submitted on 5 Jul 2021

HAL is a multi-disciplinary open access archive for the deposit and dissemination of scientific research documents, whether they are published or not. The documents may come from teaching and research institutions in France or abroad, or from public or private research centers.
L'archive ouverte pluridisciplinaire HAL, est destinée au dépôt et à la diffusion de documents scientifiques de niveau recherche, publiés ou non, émanant des établissements d'enseignement et de recherche français ou étrangers, des laboratoires publics ou privés. 


\title{
INTEGRAL REPRESENTATION AND RELAXATION OF LOCAL FUNCTIONALS ON CHEEGER-SOBOLEV SPACES
}

\author{
OMAR ANZA HAFSA AND JEAN-PHILIPPE MANDALLENA
}

\begin{abstract}
Aвstract. We prove an integral representation theorem for local functionals with polynomial growth defined on Cheeger-Sobolev spaces. More precisely, we give a version of the well-known Buttazzo-Dal maso's integral representation theorem in the framework of Cheeger-Sobolev spaces. The integral representation theorem is used to prove a relaxation theorem.
\end{abstract}

\section{INTRODUCTION}

Let $(X, d, \mu)$ be a metric measure space with $\mu$ a nontrivial Borel (regular) measure on $X$ and $(X, d)$ is a separable metric space. Let $p \in] 1, \infty[$. Cheeger showed in a seminal paper [Che99] that if we assume that $\mu$ is doubling and $(X, d, \mu)$ enjoys a $(1, p)$-Poincaré inequality (see Section 2), then $X$ has a measurable differentiable structure. That is there exists a countable collection $\left\{\left(X_{k}, \gamma^{k}\right)\right\}_{k \in \mathbb{N}}$ of measurable sets $X_{k}$ and of Lipschitz "coordinate" functions $\gamma^{k}:=\left(\gamma_{1}^{k}, \ldots, \gamma_{N(k)}^{k}\right): X_{k} \rightarrow \mathbb{R}^{N(k)}$ such that $\mu\left(X \backslash \cup_{k} X_{k}\right)=0$ and each Lipschitz function $f: X \rightarrow \mathbb{R}$ is differentiable in the sense that there exists a bounded measurable function $D_{\mu}^{k} f \in L_{\mu}^{\infty}\left(X_{k} ; \mathbb{R}^{N(k)}\right)$ such that for $\mu$-a.e. $x \in X_{k}$,

$$
\lim _{\rho \rightarrow 0} \sup _{y \in B_{\rho}(x)} \frac{\left|f(y)-f(x)-\left\langle D_{\mu}^{k} f(x), \gamma^{k}(y)-\gamma^{k}(x)\right\rangle\right|}{\rho}=0 .
$$

The Cheeger-Sobolev space $H_{\mu}^{1, p}\left(X ; \mathbb{R}^{m}\right)$ on $X$, introduced by Cheeger [Che99] (see Section 2, Definition 3), can be defined as the completion of Lipschitz functions.

Our goal is to show an integral representation and a relaxation theorem for local functionals with $p$-growth defined on Cheeger-Sobolev spaces $H_{\mu}^{1, p}\left(\Omega ; \mathbb{R}^{m}\right)$ where $\Omega \subset X$ is an open set with finite measure. More precisely, we want to give a version of the integral representation and relaxation results of [BDM85, BFLM02] in the setting of Cheeger-Sobolev spaces.

The proof of the integral representation of [BDM85] on open sets of Euclidean spaces splits into several steps, first, the integrand is defined on linear functions, which allows easily to write an integral representation on continuous piecewise affine functions. Then it is shown that necessarily the integrand is continuous with respect to the second variable (Carathéodory integrand) by proving the "zig-zag lemma" whose proof uses the lower semicontinuity property along specific construction of continuous piecewise affine functions. The conclusion comes by passing to the limit and by using the local approximation of Sobolev functions by continuous piecewise affine functions and the continuity (and the growth conditions) with respect to the second variable of the integrand. At first glance, there does not seem to be an easy way to adapt this strategy in Cheeger-Sobolev spaces.

Université de Nîmes, Laboratoire MiPA, Site des Carmes, Place Gabriel Péri, 30021 Nîmes, France

E-mail addresses: <omar .anza-hafsa@unimes.fr>, <jean-philippe.mandallena@unimes.fr $>$.

Key words and phrases. Integral representation, Relaxation, Integral functionals defined on Cheeger-Sobolev spaces. 
Especially, we do not know how to adjust the zig-zag lemma to obtain the continuity of the integrand. One way is to assume a convexity condition on the functional (see [MPSC20, MV20]).

The integral representation result of [BFLM02, Theorem 2, pp. 189] shows that the integrand can be written as limit, when the radius of balls goes to zero, of the average of minimization Dirichlet problems associated with the functional on small balls. The strategy of the proof, known as the "global relaxation method", uses mainly an intermediate representation result of an envelope, similar to the Carathéodory construction in measure theory (see Subsection 6.3), of local minimization Dirichlet problems associated with the functional. The advantage of the method is that it avoids the use of approximation by continuous piecewise affine functions. It can therefore be adapted more easily to the framework of Cheeger-Sobolev spaces, we already got several results by following this path, see [AHM15, AHM17, AHM18]. We must emphasize that this strategy makes significant use of the coercivity of the functional, which is not the case of the Buttazzo and Dal Maso's integral representation theorem [BDM85].

One motivation, for developing the calculus of variations in the setting of metric measure spaces, comes from applications to hyperelasticity. In fact, the interest of considering a general measure is that its support can be interpreted as a hyperelastic structure with its singularities like for example thin dimensions, corners, junctions, etc. Such mechanical "singular" objects naturally lead to develop calculus of variations in the setting of metric measure spaces. (We refer the reader to [BBS97, Zhi02, GJLP02] and [GPS07, Chapter $2, \S 10]$ and the references therein). Another motivation is the development of the calculus of variations on "singular" spaces, which are of interest for geometers and physicists, like Carnot groups, glued spaces, Laakso spaces, Bourdon-Pajot spaces, Gromov-Hausdorff limit spaces, spaces satisfying generalized Ricci bounds (see [KM16] for more details). Indeed, all these spaces are examples of doubling metric measure spaces satisfying a Poincaré inequality on which our integral representation and relaxation results on Cheeger-Sobolev spaces could be applied.

We assume in the following of the paper that $\mu$ is doubling, $(X, d, \mu)$ enjoys a $(1, p)$ Poincaré inequality, $(X, d)$ is a complete separable metric space, and $(X, d, \mu)$ satisfies the annular decay property (see Definition 4 ).

Throughout the rest of the paper $\Omega \subset X$ denotes an open set of finite measure $\mu(\Omega)<\infty$. We denote by $\mathcal{O}(\Omega)$ the class of all open subsets of $\Omega$.

Our first result is an integral representation theorem in Cheeger-Sobolev spaces:

Theorem 1. Let $F: H_{\mu}^{1, p}\left(\Omega ; \mathbb{R}^{m}\right) \times \mathcal{O}(\Omega) \rightarrow[0, \infty]$ satisfy

$\left(\mathrm{C}_{1}\right)$ for every $u \in H_{\mu}^{1, p}\left(\Omega ; \mathbb{R}^{m}\right)$ the set function $F(u, \cdot)$ is the restriction to $\mathcal{O}(\Omega)$ of a positive Radon measure;

$\left(\mathrm{C}_{2}\right) F(\cdot, O)$ is local, i.e. $F(u, O)=F(v, O)$ whenever $u=v \mu$-a.e. in $O$ for all $(u, v) \in$ $H_{\mu}^{1, p}\left(\Omega ; \mathbb{R}^{m}\right)^{2}$ and all $O \in \mathcal{O}(\Omega) ;$

$\left(\mathrm{C}_{3}\right) F(u+z, O)=F(u, O)$ for all $z \in \mathbb{R}^{m}$, all $u \in H_{\mu}^{1, p}\left(\Omega ; \mathbb{R}^{m}\right)$ and all $O \in \mathcal{O}(\Omega)$;

$\left(\mathrm{C}_{4}\right)$ there exist $c>0, b \geqslant 0$ and $a \in L_{\mu}^{1}(\Omega)$ such that for every $(u, O) \in H_{\mu}^{1, p}\left(\Omega ; \mathbb{R}^{m}\right) \times \mathcal{O}(\Omega)$

$$
c \int_{O}\left|\nabla_{\mu} u(x)\right|^{p} d \mu(x) \leqslant F(u, O) \leqslant \int_{O} a(x)+b\left|\nabla_{\mu} u(x)\right|^{p} d \mu(x)
$$

where $\nabla_{\mu} u$ is the $\mu$-gradient of $u$. 
$\left(\mathrm{C}_{5}\right)$ for every $O \in \mathcal{O}(\Omega)$ the functional $F(\cdot, O)$ is $L_{\mu}^{p}$-lower semicontinuous, i.e. for every $u,\left\{u_{n}\right\}_{n \in \mathbb{N}} \subset$ $L_{\mu}^{p}\left(\Omega ; \mathbb{R}^{m}\right)$ satisfying $\lim _{n \rightarrow \infty}\left\|u_{n}-u\right\|_{L_{\mu}^{p}\left(\Omega ; \mathbb{R}^{m}\right)}=0$ we have

$$
\varliminf_{n \rightarrow \infty} F\left(u_{n}, O\right) \geqslant F(u, O) \text {. }
$$

Then there exists a Borel measurable function $f: \Omega \times \mathbb{M} \rightarrow[0, \infty]$ such that

(i) for every $O \in \mathcal{O}(\Omega)$ and every $u \in H_{\mu}^{1, p}\left(\Omega ; \mathbb{R}^{m}\right)$

$$
F(u, O)=\int_{O} f\left(x, \nabla_{\mu} u(x)\right) d \mu(x)
$$

(ii) for every $k \in \mathbb{N}$, for $\mu$-a.e. $x \in \Omega \cap X_{k}$ and every $\xi \in \mathbb{M}$

$$
f(x, \xi):=\varlimsup_{\rho \rightarrow 0} \inf _{\varphi \in H_{\mu, 0}^{1, p}\left(B_{\rho}(x) ; \mathbb{R}^{m}\right)} \frac{F\left(\xi \cdot \gamma^{k}(\cdot)+\varphi, B_{\rho}(x)\right)}{\mu\left(B_{\rho}(x)\right)} ;
$$

(iii) the function $f$ is $H_{\mu}^{1, p}$-quasiconvex, i.e. for every $\xi \in \mathbb{M}$ and for $\mu$-a.e. $x \in \Omega$

$$
f(x, \xi)=\lim _{\rho \rightarrow 0} \inf _{\varphi \in H_{\mu, 0}^{1, p}\left(B_{\rho}(x) ; \mathbb{R}^{m}\right)} f_{B_{\rho}(x)} f\left(y, \xi+\nabla_{\mu} \varphi(y)\right) d \mu(y) ;
$$

(iv) for $\mu$-a.e. $x \in \Omega$ and for every $\xi \in \mathbb{M}$ we have

$$
c|\xi|^{p} \leqslant f(x, \xi) \leqslant a(x)+b|\xi|^{p}
$$

where $c>0, b \geqslant 0$ and $a \in L_{\mu}^{1}(\Omega)$ are given by $\left(\mathrm{C}_{4}\right)$ :

(v) if there exists a Borel measurable function $\tilde{f}: \Omega \times \mathbb{M} \rightarrow[0, \infty]$ such that for every $O \in \mathcal{O}(\Omega)$ and every $u \in H_{\mu}^{1, p}\left(\Omega ; \mathbb{R}^{m}\right)$

$$
F(u, O)=\int_{O} \tilde{f}\left(x, \nabla_{\mu} u(x)\right) d \mu(x)
$$

then for $\mu$-a.e. $x \in X$ and for every $\xi \in \mathbb{M}$

$$
\tilde{f}(x, \xi)=f(x, \xi) .
$$

When the functional is not necessarily $L_{\mu}^{p}$-lower semicontinuous we need to consider the $L_{\mu}^{p}$-lower semicontinuous envelope of $F(\cdot, O)$ defined by

$$
H_{\mu}^{1, p}\left(\Omega ; \mathbb{R}^{m}\right) \ni u \longmapsto \bar{F}(u, O):=\inf \left\{\varliminf_{n \rightarrow \infty} F\left(u_{n}, O\right): u_{n} \rightarrow u \operatorname{in} L_{\mu}^{p}\left(\Omega ; \mathbb{R}^{m}\right)\right\} .
$$

We have the following relaxation theorem:

Theorem 2. Let $F: H_{\mu}^{1, p}\left(\Omega ; \mathbb{R}^{m}\right) \times \mathcal{O}(\Omega) \rightarrow[0, \infty]$ satisfying $\left(\mathrm{C}_{1}\right)-\left(\mathrm{C}_{4}\right)$. Then there exists a Borel measurable function $\bar{f}: \Omega \times \mathbb{M} \rightarrow[0, \infty]$ such that

(i) for every $O \in \mathcal{O}(\Omega)$ and every $u \in H_{\mu}^{1, p}\left(\Omega ; \mathbb{R}^{m}\right)$

$$
\bar{F}(u, O)=\int_{O} \bar{f}\left(x, \nabla_{\mu} u(x)\right) d \mu(x)
$$

(ii) for every $k \in \mathbb{N}$, for $\mu$-a.e. $x \in X_{k}$ and for every $\xi \in \mathbb{M}$

$$
\bar{f}(x, \xi):=\varlimsup_{\rho \rightarrow 0} \inf _{\varphi \in H_{\mu, 0}^{1, p}\left(B_{\rho}(x) ; \mathbb{R}^{m}\right)} \frac{F\left(\xi \cdot \gamma^{k}(\cdot)+\varphi, B_{\rho}(x)\right)}{\mu\left(B_{\rho}(x)\right)} ;
$$

(iii) the function $\bar{f}$ is $H_{\mu}^{1, p}$-quasiconvex; 
(iv) for $\mu$-a.e. $x \in \Omega$ and for every $\xi \in \mathbb{M}$ we have

$$
c|\xi|^{p} \leqslant \bar{f}(x, \xi) \leqslant a(x)+b|\xi|^{p}
$$

where $c>0, b \geqslant 0$ and $a \in L_{\mu}^{1}(\Omega)$ are given by $\left(\mathrm{C}_{4}\right)$.

The following consequence of the relaxation Theorem 2 is a characterization of the lower semicontinuity of integral functionals. This is an improvement of the $H_{\mu}^{1, p}$-quasiconvexity, a necessary condition (playing the same role as the quasiconvexity concept in the Euclidean case, see for instance [BM84]) studied in [AHM20] (see Subsection 6.2).

Corollary 1. Let $f: \Omega \times \mathbb{M} \longrightarrow[0, \infty]$ be a Borel measurable function. Assume that there exists $c>0, b \geqslant 0$ and $a \in L_{\mu}^{1}(\Omega)$ such that for $\mu$-a.e. $x \in \Omega$ and for every $\xi \in \mathbb{M}$ we have

$$
c|\xi|^{p} \leqslant f(x, \xi) \leqslant a(x)+b|\xi|^{p} .
$$

The following two assertions are equivalent:

(i) for $\mu$-a.e. $x \in \Omega$ and for every $\xi \in \mathbb{M}$ it holds

$$
f(x, \xi)=\varlimsup_{\rho \rightarrow 0} \inf _{\varphi \in H_{\mu, 0}^{1, p}\left(B_{\rho}(x) ; \mathbb{R}^{m}\right)} f_{B_{\rho}(x)} f\left(y, \xi+\nabla_{\mu} \varphi\right) d \mu .
$$

(ii) for every $O \in \mathcal{O}(\Omega)$, the functional

$$
H_{\mu}^{1, p}\left(O ; \mathbb{R}^{m}\right) \ni u \longmapsto \int_{O} f\left(x, \nabla_{\mu} u(x)\right) d \mu(x)
$$

is $L_{\mu}^{p}$-lower semicontinuous.

The plan of the paper is as follows. In Section 2 we provide the materials about metric measure spaces and Cheeger-Sobolev spaces we need for our purposes. In Section 3 we give the proof of Theorem 1. The proof splits into several steps, we use first Lemma 10 which provides an integral representation of the Vitali envelope of the local minimization Dirichlet problem associated with the functional, then we can localize, using cut-off techniques, the formula of the integrand by replacing $u$ with $u_{x}(\cdot):=u(x)+\nabla_{\mu} u(x) \cdot\left(\gamma^{k}(\cdot)-\gamma^{k}(x)\right)$. In the last step, we show that the integrand is Borel measurable. In Section 4, we prove the relaxation theorem Theorem 2 with the help of Theorem 1 and by using mainly the De Giorgi-Letta Lemma which gives sufficient conditions for increasing set functions on open sets to be a measure. In Section 5 we prove Corollary 1 which is a consequence of Theorem 2 and Corollary 2 about the equality of Borel measurable integrands. This is a recast in the setting of metric measure space of an Alberti's result [Alb91]. The last Section6 is devoted to the auxiliary results we need in the proofs.

\section{Notation.}

- We will denote by $\mathcal{B}(\Omega)$ the Borel $\sigma$-algebra of $X$ and $\mathcal{B}_{\mu}(\Omega)$ the $\mu$-completion of $\mathcal{B}(\Omega)$.

- We will denote by $B_{\rho}(x):=\{y \in X: d(x, y)<\rho\}$ the open ball, and by $\bar{B}_{\rho}(x):=\{y \in$ $X: d(x, y) \leqslant \rho\}$ the closed ball, centered at $x$ with radius $\rho>0$.

- For every measurable set $A \subset \Omega$ with positive measure, and for every nonnegative measurable or integrable function $f$ on $A$, we set

$$
f_{A} f d \mu:=\frac{1}{\mu(A)} \int_{A} f(x) d \mu(x) .
$$

- The algebra of Lipschitz functions from $\Omega$ to $\mathbb{R}$ is denoted by $\operatorname{Lip}(\Omega)$. 


\section{Preliminaries: the metric measure spaces, the Cheeger-Sobolev spaces}

Let $p>1$ be a real number, let $(X, d, \mu)$ be a metric measure space, where $\mu$ is a nontrivial locally finite Borel regular measure on $X$ and $(X, d)$ is a separable metric space. In what follows, we assume that $\mu$ is doubling, i.e. there exists a constant $C_{d}$ (called doubling constant) such that

$$
\forall x \in X \quad \forall \rho>0 \quad \mu\left(B_{2 \rho}(x)\right) \leqslant C_{d} \mu\left(B_{\rho}(x)\right) .
$$

The concept of upper gradient was introduced by Heinonen and Koskela (see [HK98]), and generalized by Cheeger (see [Che99, Definition 2.8]):

\section{Definition 1.}

(i) A Borel function $g: X \rightarrow[0, \infty]$ is said to be an upper gradient for $f: X \rightarrow \mathbb{R}$ if

$$
|f(c(1))-f(c(0))| \leqslant \int_{0}^{1} g(c(s)) d s
$$

for all continuous rectifiable curves $c:[0,1] \rightarrow X$.

(ii) A function $g \in L_{\mu}^{p}(X)$ is said to be a p-weak upper gradient for $f \in L_{\mu}^{p}(X)$ if there exist $\left\{f_{n}\right\}_{n} \subset L_{\mu}^{p}(X)$ and $\left\{g_{n}\right\}_{n} \subset L_{\mu}^{p}(X)$ such that for each $n \geqslant 1, g_{n}$ is an upper gradient for $f_{n}, f_{n} \rightarrow f$ in $L_{\mu}^{p}(X)$ and $g_{n} \rightarrow g$ in $L_{\mu}^{p}(X)$.

Definition 2. The metric measure space $(X, d, \mu)$ enjoys a $(1, p)$-Poincaré inequality with $p \in$ ]1, $\infty$ [ if there exist $\mathfrak{C}_{p}>0$ and $\sigma \geqslant 1$ such that for every $x \in X$ and every $\rho>0$,

$$
f_{B_{\rho}(x)}\left|f(y)-f_{B_{\rho}(x)} f d \mu\right| d \mu(y) \leqslant \rho \mathfrak{C}_{p}\left(f_{B_{\sigma \rho}(x)} g^{p} d \mu\right)^{\frac{1}{p}}
$$

for every $f \in L_{\mu}^{p}(X)$ and every $p$-weak upper gradient $g \in L_{\mu}^{p}(X)$ for $f$.

From Cheeger [Che99, Theorem 4.38] (see also Keith [Kei04, Definition 2.1.1 and Theorem 2.3.1]) we have:

Theorem 3. If $\mu$ is doubling, i.e. (4) holds, and $X$ enjoys a $(1, p)$-Poincaré inequality, i.e. (5) holds, then there exist a countable family $\left\{\left(X_{k}, \gamma^{k}\right)\right\}_{k \in \mathbb{N}}$ of $\mu$-measurable disjoint subsets $X_{k}$ of $X$ with $\mu\left(X \backslash \cup_{k=0}^{\infty} X_{k}\right)=0$ and of functions $\gamma^{k}=\left(\gamma_{1}^{k}, \cdots, \gamma_{N(k)}^{k}\right): X \rightarrow \mathbb{R}^{N(k)}$ with $\gamma_{i}^{k} \in \operatorname{Lip}(X)$ satisfying the following properties:

(i) there exists an integer $N \geqslant 1$ such that $N(k) \in\{1, \ldots, N\}$ for all $k \in \mathbb{N}$;

(ii) for every $k \in \mathbb{N}$ and every $f \in \operatorname{Lip}(X)$ there is a unique $D_{\mu}^{k} f \in L_{\mu}^{\infty}\left(X_{k} ; \mathbb{R}^{N(k)}\right)$ such that for $\mu$-a.e. $x \in X_{k}$,

$$
\lim _{\rho \rightarrow 0} \sup _{y \in B_{\rho}(x)} \frac{\left|f(y)-f(x)-\left\langle D_{\mu}^{k} f(x), \gamma^{k}(y)-\gamma^{k}(x)\right\rangle\right|}{\rho}=0,
$$

where $f_{x} \in \operatorname{Lip}(X)$ is given by $f_{x}(y):=f(x)+\left\langle D_{\mu}^{k} f(x), \gamma^{k}(y)-\gamma^{k}(x)\right\rangle$; in particular

$$
D_{\mu}^{k} f_{x}(y)=D_{\mu}^{k} f(x) \text { for } \mu \text {-a.a. } y \in X_{k}
$$

(iii) the operator $D_{\mu}: \operatorname{Lip}(X) \rightarrow L_{\mu}^{\infty}\left(X ; \mathbb{R}^{N}\right)$ given by

$$
D_{\mu} f:=\sum_{k \in \mathbb{N}} \mathbb{1}_{X_{k}} D_{\mu}^{k} f
$$

where $\mathbb{1}_{X_{k}}$ denotes the characteristic function of $X_{k}$, is linear and, for each $f, g \in \operatorname{Lip}(X)$, we have

$$
D_{\mu}(f g)=\underset{5}{f} D_{\mu} g+g D_{\mu} f
$$


(iv) for every $f \in \operatorname{Lip}(X), D_{\mu} f=0 \mu$-a.e. on every $\mu$-measurable set where $f$ is constant.

We set $\mathbb{M}=\mathbb{R}^{m \times N}$ where $N$ is given by Theorem 3 (i), For every $k \in \mathbb{N}$ we denote by $|\cdot|_{k}$ the Euclidean norm on $\mathbb{R}^{m \times N(k)}$ where $N(k)$ is given by Theorem 3 , and we set for every $x \in \Omega$ and every $\xi \in \mathbb{M}$

$$
|\xi|_{x}:=\sum_{k=0}^{\infty}|\xi|_{k} \mathbb{1}_{X_{k}}(x) .
$$

There exists Borel sets $\Omega \supset B_{k} \supset X_{k} \cap \Omega$ such that $\mu\left(\Omega \backslash\left(\cup_{k=0}^{\infty} B_{k}\right)\right)=0$ since the regularity of $\mu$. If we define $N: \Omega \times \mathbb{M} \rightarrow \mathbb{R}_{+}$by

$$
N(x, \xi):=\sum_{k=0}^{\infty}|\xi|_{k} \mathbb{1}_{B_{k}}(x),
$$

then we can see that $N$ is Borel measurable and coincide with $\mid \cdot{ }_{x}$ for $\mu$-a.e. $x \in \Omega$.

Lemma 1. There exists a Borel measurable function $N: \Omega \times \mathbb{M} \rightarrow \mathbb{R}_{+}$such that for $\mu$-a.e. in $\Omega$ and for every $\xi \in \mathrm{M}$ we have $N(x, \xi)=|\xi|_{x}$.

In the following, for every $k \in \mathbb{N}$ and every $x \in X_{k} \cap \Omega$, we simply denote the norm of $\xi \in \mathbb{R}^{N(k) \times m} \subset \mathbb{M}$ by $|\xi|$ instead of $|\xi|_{x}$.

Let $\operatorname{Lip}\left(\Omega ; \mathbb{R}^{m}\right):=[\operatorname{Lip}(\Omega)]^{m}$ and let $\nabla_{\mu}: \operatorname{Lip}\left(\Omega ; \mathbb{R}^{m}\right) \longrightarrow L_{\mu}^{\infty}(\Omega ; \mathbb{M})$ given by

$$
\nabla_{\mu} u:=\left(\begin{array}{c}
D_{\mu} u_{1} \\
\vdots \\
D_{\mu} u_{m}
\end{array}\right) \text { with } u=\left(u_{1}, \cdots, u_{m}\right) .
$$

From Theorem 3 (iii) we see that for every $u \in \operatorname{Lip}\left(\Omega ; \mathbb{R}^{m}\right)$ and every $f \in \operatorname{Lip}(\Omega)$, we have

$$
\nabla_{\mu}(f u)=f \nabla_{\mu} u+D_{\mu} f \otimes u .
$$

Definition 3. The $p$-Cheeger-Sobolev space $H_{\mu}^{1, p}\left(\Omega ; \mathbb{R}^{m}\right)$ is defined as the completion of the space of Lipschitz functions $\operatorname{Lip}\left(\Omega ; \mathbb{R}^{m}\right)$ with respect to the norm

$$
\|u\|_{H_{\mu}^{1, p}\left(\Omega ; \mathbb{R}^{m}\right)}:=\|u\|_{L_{\mu}^{p}\left(\Omega ; \mathbb{R}^{m}\right)}+\left\|\nabla_{\mu} u\right\|_{L_{\mu}^{p}(\Omega ; \mathbb{M})} .
$$

Taking Proposition 1 (ii) below into account, since $\left\|\nabla_{\mu} u\right\|_{L_{\mu}^{p}(\Omega ; \mathbb{M})} \leqslant\|u\|_{H_{\mu}^{1, p}\left(\Omega ; \mathbb{R}^{m}\right)}$ for all $u \in \operatorname{Lip}\left(\Omega ; \mathbb{R}^{m}\right)$ the linear map $\nabla_{\mu}$ from $\operatorname{Lip}\left(\Omega ; \mathbb{R}^{m}\right)$ to $L_{\mu}^{p}(\Omega ; \mathbb{M})$ has a unique extension to $H_{\mu}^{1, p}\left(\Omega ; \mathbb{R}^{m}\right)$ which will still be denoted by $\nabla_{\mu}$ and will be called the $\mu$-gradient.

Remark 1. In fact, the original Cheeger's definition [Che99, (2.1), pp. 440] of $H_{\mu}^{1, p}(\Omega)$ is all the functions $u \in L_{\mu}^{p}(\Omega)$ for which $|u|_{1, p}<\infty$ where

$$
|u|_{1, p}=|u|_{L_{\mu}^{p}(\Omega)}+\inf \left\{\varliminf_{n \rightarrow \infty}\left|g_{u_{n}}\right|_{L_{\mu}^{p}(\Omega)}: L_{\mu}^{p}(\Omega) \ni u_{n} \rightarrow u \text { in } L_{\mu}^{p}\right\}
$$

where $g_{u_{n}}$ is an upper gradient for $u_{n}$ for all $n \in \mathbb{N}$. Cheeger [Che99, Theorem 4.47, pp. 459] shows that the space $H_{\mu}^{1, p}(\Omega)$ can be seen as the completion of Lipschitz functions for the norm $|u|_{L_{\mu}^{p}(\Omega)}+\left|D_{\mu} u\right|_{L_{\mu}^{p}(\Omega)}$ for all $u \in \operatorname{Lip}(\Omega)$. This latter norm coincide with $|\cdot|_{1, p}$ by combining [Che99, Definition 2.9 and Theorem 2.10, pp. 441] and [Che99, Corollary 4.41, pp. 458].

For more details on the various possible extensions of the classical theory of the Sobolev spaces to the setting of metric measure spaces, we refer to [Hei07, 10-14] (see also [Che99, Sha00, GT01, Haj03]).

The following proposition gathers some results of many authors and provides useful properties for dealing with calculus of variations in the metric measure setting. 
Proposition 1. Under the hypotheses of Theorem 3, we have:

(i) (from [HKST15, Theorem 3.4.3, pp. 73]) the metric measure space X satisfies the Vitali covering theorem, i.e. for every $A \subset X$ and every family $\mathcal{F}$ of closed balls in $X$, if $\inf \left\{\rho>0: \bar{B}_{\rho}(x) \in \mathcal{F}\right\}=0$ for all $x \in A$ then there exists a countable disjoint subfamily $\mathcal{G}$ of $\mathcal{F}$ such that $\mu\left(A \backslash \cup_{B \in \mathcal{G}} B\right)=0$; in other words, $A \subset\left(\cup_{B \in \mathcal{G}} B\right) \cup N$ with $\mu(N)=0$;

(ii) (from [FHK99, Theorem 10]) the $\mu$-gradient is closable in $H_{\mu}^{1, p}\left(\Omega ; \mathbb{R}^{m}\right)$, i.e. for every $u \in H_{\mu}^{1, p}\left(\Omega ; \mathbb{R}^{m}\right)$ and every measurable set $A \subset \Omega$, if $u(x)=0$ for $\mu$-a.a. $x \in A$ then $\nabla_{\mu} u(x)=0$ for $\mu$-a.a. $x \in A$;

(iii) (from [BB11, Corollary 4.24 pp. 93], [BB11, Theorem 5.51, pp. 142] and [Che99, $\S 4])$ the metric space $X$ enjoys a $p$-Sobolev inequality, i.e. there exists $C_{S}>0$ such that

$$
\left(\int_{B_{\rho}(x)}|v|^{p} d \mu\right)^{\frac{1}{p}} \leqslant \rho C_{S}\left(\int_{B_{\rho}(x)}\left|\nabla_{\mu} v\right|^{p} d \mu\right)^{\frac{1}{p}}
$$

for all $0<\rho \leqslant \rho_{0}$, with $\rho_{0}>0$, and all $v \in H_{\mu, 0}^{1, p}\left(B_{\rho}(x) ; \mathbb{R}^{m}\right)$, where, for each $O \in \mathcal{O}(X)$, $H_{\mu, 0}^{1, p}\left(O ; \mathbb{R}^{m}\right)$ is the closure of $\operatorname{Lip}_{0}\left(O ; \mathbb{R}^{m}\right)$ with respect to $H_{\mu}^{1, p}$-norm defined in $(8)$ with

$$
\operatorname{Lip}_{0}\left(O ; \mathbb{R}^{m}\right):=\left\{u \in \operatorname{Lip}\left(X ; \mathbb{R}^{m}\right): u=0 \text { on } X \backslash O\right\} ;
$$

(iv) (from [Bjö00, Theorem 4.5 and Corollary 4.6] or [GH13, Theorem 2.12]) for every $u \in H_{\mu}^{1, p}\left(\Omega ; \mathbb{R}^{m}\right)$ and $\mu$-a.e. $x \in \Omega$ there exists $u_{x} \in H_{\mu}^{1, p}\left(\Omega ; \mathbb{R}^{m}\right)$ given by $u_{x}(y):=$ $u(x)+\nabla_{\mu} u(x) \cdot\left(\gamma^{k}(y)-\gamma^{k}(x)\right)$ such that

$$
\begin{array}{r}
\nabla_{\mu} u_{x}(y)=\nabla_{\mu} u(x) \text { for } \mu \text {-a.a. } y \in X ; \\
\lim _{\rho \rightarrow 0} \frac{1}{\rho}\left(f_{B_{\rho}(x)}\left|u(y)-u_{x}(y)\right|^{p} d \mu(y)\right)^{\frac{1}{p}}=0 ;
\end{array}
$$

(v) (from [Che99, Theorem 6.1]) for every $x \in \Omega$, every $\rho>0$ and every $\tau \in] 0,1[$ there exists a function $\varphi \in \operatorname{Lip}(\Omega ;[0,1])$ such that

$$
\varphi(x)=0 \text { for all } x \in \Omega \backslash B_{\rho}(x), \varphi(x)=1 \text { for all } x \in \bar{B}_{\tau \rho}(x)
$$

and

$$
\left\|D_{\mu} \varphi\right\|_{L_{\mu}^{\infty}\left(\Omega ; \mathbb{R}^{N}\right)} \leqslant \frac{C_{0}}{\rho(1-\tau)} \text { for some } C_{0}>0 .
$$

Such $a \varphi$ is called an Urysohn function for the pair $\left(\Omega \backslash B_{\rho}(x), \bar{B}_{\tau \rho}(x)\right)$.

Definition 4 . We say that the metric measure space $(X, d, \mu)$ satisfies the annular decay property if there exist $\eta>0$ and $K_{0} \geqslant 1$ such that for every $x \in X$, every $\rho>0$ and every $\left.\tau \in\right] 0,1[$,

$$
\mu\left(B_{\rho}(x) \backslash B_{\tau \rho}(x)\right) \leqslant K_{0}(1-\tau)^{\eta} \mu\left(B_{\rho}(x)\right) .
$$

The annular decay property was introduced independently by [Buc99, pp. 521 and $\S 2$ pp. 524] and [CM98]. This property holds, for instance, when the metric space is a length space, i.e. metric space in which the distance between points is the infimum of lenghts of rectifiable paths joining those points, see [Buc99, Corollary 2.2], [CM98, Lemma 3.3], [Che99, Proposition 6.12] and [HKST15, Proposition 11.5.3, pp. 328].

Remark 2. We can remark that, when the annular decay property holds, the boundary of balls is of zero measure, indeed, if $x \in X, \rho>0$ and $\tau \in] \frac{1}{2}, 1[$, we have

$$
\mu\left(\partial B_{\rho}(x)\right) \leqslant \mu\left(B_{\frac{\rho}{\tau}}(x) \backslash B_{\rho}(x)\right) \leqslant K_{0}(1-\tau)^{\eta} \mu\left(B_{\frac{\rho}{\tau}}(x)\right) \leqslant K_{0}(1-\tau)^{\eta} \mu\left(B_{2 \rho}(x)\right),
$$

letting $\tau \rightarrow 1$ we obtain that $\mu\left(\partial B_{\rho}(x)\right)=0$. 
Lemma 2. Assume that the annular decay property holds. Let $\lambda$ be a positive Radon measure. Let $x \in X$ satisfy

$$
\lim _{\rho \rightarrow 0} \frac{\lambda\left(B_{\rho}(x)\right)}{\mu\left(B_{\rho}(x)\right)}=: \frac{d \lambda}{d \mu}(x)<\infty
$$

Then for every $\tau \in] 0,1[$

$$
\varlimsup_{\rho \rightarrow 0} \frac{\lambda\left(B_{\rho}(x) \backslash B_{\tau \rho}(x)\right)}{\mu\left(B_{\rho}(x)\right)} \leqslant K_{0}(1-\tau)^{\eta} \frac{d \lambda}{d \mu}(x) .
$$

In particular, we have

$$
\lim _{\tau \rightarrow 1} \varlimsup_{\rho \rightarrow 0} \frac{\lambda\left(B_{\rho}(x) \backslash B_{\tau \rho}(x)\right)}{\mu\left(B_{\rho}(x)\right)}=0 .
$$

Proof of Lemma 2, Let $x \in X$ satisfy (11). We can write for every $\rho>0$

$$
\begin{aligned}
\frac{\lambda\left(B_{\rho}(x) \backslash B_{\tau \rho}(x)\right)}{\mu\left(B_{\rho}(x)\right)} & =\frac{\lambda\left(B_{\rho}(x)\right)}{\mu\left(B_{\rho}(x)\right)}-\frac{\mu\left(B_{\tau \rho}(x)\right)}{\mu\left(B_{\rho}(x)\right)} \frac{\lambda\left(B_{\tau \rho}(x)\right)}{\mu\left(B_{\tau \rho}(x)\right)} \\
& =\left(\frac{\lambda\left(B_{\rho}(x)\right)}{\mu\left(B_{\rho}(x)\right)}-\frac{\lambda\left(B_{\tau \rho}(x)\right)}{\mu\left(B_{\tau \rho}(x)\right)}\right)+\frac{\mu\left(B_{\rho}(x) \backslash B_{\tau \rho}(x)\right)}{\mu\left(B_{\rho}(x)\right)} \frac{\lambda\left(B_{\tau \rho}(x)\right)}{\mu\left(B_{\tau \rho}(x)\right)} \\
& \leqslant\left(\frac{\lambda\left(B_{\rho}(x)\right)}{\mu\left(B_{\rho}(x)\right)}-\frac{\lambda\left(B_{\tau \rho}(x)\right)}{\mu\left(B_{\tau \rho}(x)\right)}\right)+K_{0}(1-\tau)^{\eta} \frac{\lambda\left(B_{\tau \rho}(x)\right)}{\mu\left(B_{\tau \rho}(x)\right)},
\end{aligned}
$$

letting $\rho \rightarrow 0$ and using (11), we obtain (12)

We will need a version of the Rellich-Kondrachov theorem in the metric measure spaces setting. The following result is due to [HK00, Theorem 8.1, pp. 37] (see also [Kał99]).

Theorem 4. Let $B \subset \Omega$ a ball. Let $\left\{\varphi_{n}\right\}_{n \in \mathbb{N}} \subset H_{\mu}^{1, p}\left(B ; \mathbb{R}^{m}\right)$ be a bounded sequence, i.e.

$$
\sup _{n \in \mathbb{N}}\left(\int_{B}\left|\varphi_{n}\right|^{p} d \mu\right)^{\frac{1}{p}}+\left(\int_{B}\left|\nabla_{\mu} \varphi_{n}\right|^{p} d \mu\right)^{\frac{1}{p}}<\infty .
$$

Then there exist a subsequence $\left\{\varphi_{n_{i}}\right\}_{i \in \mathbb{N}} \subset H_{\mu}^{1, p}\left(B ; \mathbb{R}^{m}\right)$ and $\varphi \in H_{\mu}^{1, p}\left(B ; \mathbb{R}^{m}\right)$ such that

$$
\lim _{i \rightarrow \infty} \int_{B}\left|\varphi_{n_{i}}-\varphi\right|^{p} d \mu=0 .
$$

Outline of the proof of Theorem 4. For the sake of simplicity we assume that $m=1$. By a development of Cheeger [Che99, pp. 449-450], we have, in our setting (doubling measure and Poincaré inequality), for every $u \in H_{\mu}^{1, p}(X)$, every $x \in X$ and every $r>0$ the following Poincaré-Sobolev inequality

$$
\left(f_{B_{r}(x)}\left|u(y)-f_{B_{r}(x)} u d \mu\right|^{\chi p} d \mu(y)\right)^{\frac{1}{\chi p}} \leqslant C r\left(f_{B_{r}(x)}\left|D_{\mu} u\right|^{p} d \mu(y)\right)^{\frac{1}{p}}
$$

for some $\chi>1$ which only depends on the doubling constant $C_{d}$ in (4) and the constant $\mathfrak{C}_{p}$ in (5), and for some $C>0$ depending on the constants $\mathfrak{C}_{p}, C_{d}$ and on the constant $\sigma$ appearing in (5).

Consider a ball $B=B_{\bar{\rho}}(\bar{x}) \subset \Omega$ and a bounded sequence $\left\{\varphi_{n}\right\}_{n \in \mathbb{N}} \subset H_{\mu}^{1, p}\left(B ; \mathbb{R}^{m}\right)$.

From (13), we have for every $n \in \mathbb{N}$

$$
\left\|\varphi_{n}-f_{B} \varphi_{n} d \mu\right\|_{L_{\mu}^{\chi p}(B)} \leqslant C \mu(B)^{\frac{1}{\chi p}-\frac{1}{p}} \bar{\rho}\left\|D_{\mu} \varphi_{n}\right\|_{L_{\mu}^{p}(B)}
$$


thus

$$
\left\|\varphi_{n}\right\|_{L_{\mu}^{\chi p}(B)} \leqslant \mu(B)^{\frac{1}{\chi p}}\left|f_{B} \varphi_{n} d \mu\right|+C \mu(B)^{\frac{1}{\chi p}-\frac{1}{p}} \bar{\rho}\left\|D_{\mu} \varphi_{n}\right\|_{L_{\mu}^{p}(B)},
$$

and by using the Hölder inequality we obtain

$$
\left\|\varphi_{n}\right\|_{L_{\mu}^{\chi p}(B)} \leqslant \mu(B)^{\frac{1}{\chi p}-\frac{1}{p}}\left(\left\|\varphi_{n}\right\|_{L_{\mu}^{p}(B)}+C \bar{\rho}\left\|D_{\mu} \varphi_{n}\right\|_{L_{\mu}^{p}(B)}\right)
$$

which means that the sequence $\left\{\varphi_{n}\right\}_{n \in \mathbb{N}} \subset L_{\mu}^{\chi p}(B)$ is bounded. Thus, there exists a subsequence (not relabelled) which weakly converges in $L_{\mu}^{\chi p}(B)$ to some $\varphi \in L_{\mu}^{\chi p}(B)$.

Lemma 3. [HK00, Lemma 8.2, pp. 37] Let $B^{\prime} \subset B$ be a ball and $\chi>1$. Let $\left\{\psi_{n}\right\}_{n \in \mathbb{N}} \subset$ $L_{\mu}^{\chi p}\left(B^{\prime}\right)$ be a bounded sequence. If $\left\{\psi_{n}\right\}_{n \in \mathbb{N}}$ converges in measure to $\psi \in L_{\mu}^{\chi p}\left(B^{\prime}\right)$ then

$$
\lim _{n \rightarrow \infty}\left\|\psi_{n}-\psi\right\|_{L_{\mu}^{p}\left(B^{\prime}\right)}=0 .
$$

Taking Lemma 3 into account, we see that it suffices to show that $\left\{\varphi_{n}\right\}_{n \in \mathbb{N}}$ converges in measure to $\varphi$.

Let $t \in] 0,1\left[\right.$. Fix $\varepsilon>0$ and $n \in \mathbb{N}$. We set for every positive $r<\frac{\bar{\rho}(1-t)}{\sigma}$ (where $\sigma$ is the constant appearing in (5)) and every $x \in t B:=B_{t \bar{\rho}}(\bar{x})$

$$
\varphi_{r}(x):=f_{B_{r}(x)} \varphi d \mu \quad \text { and } \quad \varphi_{n, r}(x):=f_{B_{r}(x)} \varphi_{n} d \mu .
$$

We have

$$
\begin{aligned}
\mu\left(t B \cap\left[\left|\varphi_{n}-\varphi\right|>\varepsilon\right]\right) \leqslant & \mu\left(t B \cap\left[\left|\varphi_{n}-\varphi_{n, r}\right|>\frac{\varepsilon}{3}\right]\right)+\mu\left(t B \cap\left[\left|\varphi_{n, r}-\varphi_{r}\right|>\frac{\varepsilon}{3}\right]\right) \\
& +\mu\left(t B \cap\left[\left|\varphi_{r}-\varphi\right|>\frac{\varepsilon}{3}\right]\right)
\end{aligned}
$$

Since the Lebesgue differentiation theorem, $\mu\left(t B \cap\left[\left|\varphi_{r}-\varphi\right|>\frac{\varepsilon}{3}\right]\right)$ goes to 0 as $r \rightarrow 0$. The term $\mu\left(t B \cap\left[\left|\varphi_{n, r}-\varphi_{r}\right|>\frac{\varepsilon}{3}\right]\right)$ tends to 0 as $n \rightarrow \infty$ for all $r>0$, since the weak convergence of $\left\{\varphi_{n}\right\}_{n \in \mathbb{N}}$ in $L_{\mu}^{\chi p}(B)$ to $\varphi$. Using [HK00, Proof of theorem 3.2, pp. 13-14] we can deduce for every $x \in t B$

$$
\left|\varphi_{n}(x)-\varphi_{n, r}(x)\right| \leqslant K r\left(\sup _{\rho \in] 0, \sigma r[} f_{B_{\rho}(x)}\left|D_{\mu} \varphi_{n}\right|^{p} d \mu\right)^{\frac{1}{p}} \leqslant K r\left(\sup _{r>0} f_{B_{r}(x) \cap B}\left|D_{\mu} \varphi_{n}\right|^{p} d \mu\right)^{\frac{1}{p}}
$$

for some constant $K>0$ depending only on the doubling constant and the constant $\mathfrak{C}_{p}$ appearing in the Poincaré inequality (5). Using the maximal theorem [HK00, Theorem 14.13], there exists $C^{\prime}>0$ depending on the doubling constant only such that

$$
\begin{aligned}
\mu\left(t B \cap\left[\left|\varphi_{n}-\varphi_{n, r}\right|>\frac{\varepsilon}{3}\right]\right) & \leqslant \mu\left(\left[\sup _{r>0} f_{B_{r}(\bullet) \cap B}\left|D_{\mu} \varphi_{n}\right|^{p} d \mu>\left(\frac{1}{3 K}\right)^{p}\left(\frac{\varepsilon}{r}\right)^{p}\right]\right) \\
& \leqslant C^{\prime}(3 K)^{p}\left(\frac{r}{\varepsilon}\right)^{p} \sup _{n \in \mathbb{N}} \int_{B}\left|D_{\mu} \varphi_{n}\right|^{p} d \mu,
\end{aligned}
$$

therefore $\sup _{n \in \mathbb{N}} \mu\left(t B \cap\left[\left|\varphi_{n}-\varphi_{n, r}\right|>\frac{\varepsilon}{3}\right]\right)$ tends to 0 as $r \rightarrow 0$. It follows that

$$
\lim _{n \rightarrow \infty} \mu\left(t B \cap\left[\left|\varphi_{n}-\varphi\right|>\varepsilon\right]\right)=0 .
$$

Applying Lemma 3 with $\psi_{n}=\varphi_{n}\left\lfloor_{t B}, \psi=\varphi\left\lfloor_{t B}\right.\right.$ and $B^{\prime}=t B$, we obtain

$$
\lim _{n \rightarrow \infty}\left\|\varphi_{n}-\varphi\right\|_{L_{\mu}^{p}(t B)}=0 \text {. }
$$


Now, since the sequence $\left\{\varphi_{n}\right\}_{n \in \mathbb{N}}$ is bounded in $L_{\mu}^{\chi p}(B)$, we have $\sup _{n \in \mathbb{N}}\left\|\varphi_{n}-\varphi\right\|_{L_{\mu}^{\chi p}(B)}<$ $\infty$. Choose $\left.t_{\varepsilon} \in\right] 0,1\left[\right.$ such that $\mu\left(B \backslash t_{\varepsilon} B\right) \leqslant \varepsilon^{\frac{\chi}{\chi^{-1}}}\left(1+\sup _{n \in \mathbb{N}}\left\|\varphi_{n}-\varphi\right\|_{L_{\mu}^{\chi p}(B)}^{p}\right)^{-1}$, by the Hölder inequality we have

$$
\begin{aligned}
\int_{B}\left|\varphi_{n}-\varphi\right|^{p} d \mu & =\int_{t_{\varepsilon} B}\left|\varphi_{n}-\varphi\right|^{p} d \mu+\int_{B \backslash t_{\varepsilon} B}\left|\varphi_{n}-\varphi\right|^{p} d \mu \\
& \leqslant\left\|\varphi_{n}-\varphi\right\|_{L_{\mu}^{p}\left(t_{\varepsilon} B\right)}^{p}+\left\|\varphi_{n}-\varphi\right\|_{L_{\mu}^{\chi p}(B)}^{p} \mu\left(B \backslash t_{\varepsilon} B\right)^{\frac{\chi-1}{\chi}} \\
& \leqslant\left\|\varphi_{n}-\varphi\right\|_{L_{\mu}^{p}\left(t_{\varepsilon} B\right)}+\varepsilon
\end{aligned}
$$

letting $n \rightarrow \infty$ and then $\varepsilon \rightarrow 0$ we obtain the desired result.

\section{Proof of Theorem 1}

For a functional $F: H_{\mu}^{1, p}\left(\Omega ; \mathbb{R}^{m}\right) \times \mathcal{O}(\Omega) \rightarrow[0, \infty]$ we define $m: H_{\mu}^{1, p}\left(\Omega ; \mathbb{R}^{m}\right) \times \mathcal{O}(\Omega) \rightarrow$ $[0, \infty]$ by

$$
m(u, O):=\inf \left\{F(u+\varphi, O): \varphi \in H_{\mu, 0}^{1, p}\left(O ; \mathbb{R}^{m}\right)\right\} .
$$

\subsection{Proof of (i) and (ii),}

Step 1: integral representation of $\boldsymbol{F}(\boldsymbol{u}, \cdot)$ via $\boldsymbol{m}(\boldsymbol{u}, \cdot)$. In this step, we show that for every $u \in H_{\mu}^{1, p}\left(\Omega ; \mathbb{R}^{m}\right)$ and every $O \in \mathcal{O}(\Omega)$

$$
F(u, O)=\int_{O} \lim _{\rho \rightarrow 0} \frac{m\left(u, B_{\rho}(x)\right)}{\mu\left(B_{\rho}(x)\right)} d \mu(x) .
$$

Let $(u, O) \in H_{\mu}^{1, p}\left(\Omega ; \mathbb{R}^{m}\right) \times \mathcal{O}(\Omega)$. By lemma 10 we have

$$
m_{-}^{*}(u, O)=\int_{O} \lim _{\rho \rightarrow 0} \frac{m\left(u, B_{\rho}(x)\right)}{\mu\left(B_{\rho}(x)\right)} d \mu(x),
$$

where

$$
m_{-}^{*}(u, O):=\sup _{\varepsilon>0} \inf \left\{\sum_{i \in I} m\left(u, B_{i}\right):\left\{B_{i}\right\}_{i \in I} \in \mathcal{V}^{\varepsilon}(O)\right\}
$$

with for every $\varepsilon>0$

$$
\begin{aligned}
\mathcal{V}^{\varepsilon}(O):=\left\{\left\{B_{i}\right\}_{i \in I} \subset \mathfrak{B}(\Omega): I\right. & \text { is countable, } \mu\left(O \backslash \cup_{i \in I} B_{i}\right)=0, \bar{B}_{i} \subset O, \\
& \left.0<\operatorname{diam}\left(B_{i}\right) \leqslant \varepsilon \text { and } \bar{B}_{i} \cap \bar{B}_{j}=\varnothing \text { for all } i \neq j\right\} .
\end{aligned}
$$

By $\left(\mathrm{C}_{1}\right)$ and $\left(\mathrm{C}_{4}\right)$, we see that $F(u, \cdot)$ is a positive Radon measure which is absolutely continuous with respect to $\mu$, so, we have

$$
m_{-}^{*}(u, O) \leqslant F(u, O) .
$$

It remains to prove that

$$
F(u, O) \leqslant m_{-}^{*}(u, O) .
$$

Fix $\varepsilon>0$. There exists a countable family of mutually disjoints balls $\left\{B_{i}\right\}_{i \in I} \in \mathcal{V}_{\varepsilon}(O)$ such that

$$
\sum_{i \in I} m\left(u, B_{i}\right) \leqslant m_{-}^{*}(u, O)+\frac{\varepsilon}{2}
$$

Given any $i \in I$, by definition of $m\left(u, B_{i}\right)$, there exists $\varphi_{\varepsilon}^{i} \in H_{\mu, 0}^{1, p}\left(B_{i} ; \mathbb{R}^{m}\right)$ such that

$$
F\left(u+\varphi_{\varepsilon}^{i}, B_{i}\right) \leqslant m\left(u, B_{i}\right)+\varepsilon \frac{\mu\left(B_{i}\right)}{2 \mu(O)} .
$$


Define $\varphi_{\varepsilon}: \Omega \rightarrow \mathbb{R}^{m}$ by $\varphi_{\varepsilon}:=\sum_{i=0}^{\infty} \varphi_{\varepsilon}^{i} \mathbb{1}_{B_{i}} \in H_{\mu, 0}^{1, p}\left(O ; \mathbb{R}^{m}\right)$, that is

$$
\varphi_{\varepsilon}:= \begin{cases}0 & \text { in } \Omega \backslash O \\ \varphi_{\varepsilon}^{i} & \text { in } B_{i} .\end{cases}
$$

Take the sum over the countable family of mutually disjoints balls $\left\{B_{i}\right\}_{i \in I}$ in (17), we obtain by using $\left(\mathrm{C}_{2}\right)$ and $\left(\mathrm{C}_{1}\right)$

$$
F\left(u+\varphi_{\varepsilon}, O\right)=\sum_{i \in I} F\left(u+\varphi_{\varepsilon}, B_{i}\right)=\sum_{i \in I} F\left(u+\varphi_{\varepsilon}^{i}, B_{i}\right) \leqslant \sum_{i \in I} m\left(u, B_{i}\right)+\frac{\varepsilon}{2} \leqslant m_{-}^{*}(u, O)+\varepsilon .
$$

If $\lim _{\varepsilon \rightarrow 0}\left\|\varphi_{\varepsilon}\right\|_{L_{\mu}^{p}\left(X ; \mathbb{R}^{m}\right)}=0$ then we get 16$)$ by using $\left(\mathrm{C}_{1}\right)$ the lower semicontinuity of $F(\cdot, O)$. So, it remains to prove that $\varphi_{\varepsilon} \rightarrow 0$ in $L_{\mu}^{p}\left(\Omega ; \mathbb{R}^{m}\right)$ as $\varepsilon \rightarrow 0$. We have by using the Sobolev inequality $[9]$, the coercivity condition $\left(\mathrm{C}_{4}\right),(14)$ and the growth condition $\left(\mathrm{C}_{4}\right)$

$$
\begin{aligned}
\int_{X}\left|\varphi_{\varepsilon}\right|^{p} d \mu=\int_{O}\left|\varphi_{\varepsilon}\right|^{p} d \mu=\sum_{i \in I} \int_{B_{i}}\left|\varphi_{k}^{i}\right|^{p} d \mu & \leqslant \sum_{i \in I} \varepsilon^{p} C_{S}^{p} \int_{B_{i}}\left|\nabla_{\mu} \varphi_{\varepsilon}^{i}\right|^{p} d \mu \\
& \leqslant \frac{2^{p-1} C_{S}^{p} \varepsilon^{p}}{c} \sum_{i \in I} F\left(u+\varphi_{\varepsilon}^{i}, B_{i}\right)+F\left(u, B_{i}\right) \\
& \leqslant \frac{2^{p-1} C_{S}^{p} \varepsilon^{p}}{c}\left(m_{-}^{*}(u, O)+\varepsilon+F(u, O)\right) \\
& \leqslant \frac{2^{p} C_{S}^{p} \varepsilon^{p}}{c}(\varepsilon+F(u, O)) \\
& \leqslant \frac{2^{p} C_{S}^{p} \varepsilon^{p}}{c}\left(\varepsilon+\int_{O} a(x)+b\left|\nabla_{\mu} u(x)\right|^{p} d \mu(x)\right)
\end{aligned}
$$

by passing to the limit $\varepsilon \rightarrow 0$ we find that $\varphi_{\varepsilon} \rightarrow 0$ in $L_{\mu}^{p}\left(X ; \mathbb{R}^{m}\right)$. Thus we obtain

$$
F(u, O)=m_{-}^{*}(u, O)=\int_{O} \lim _{\rho \rightarrow 0} \frac{m\left(u, B_{\rho}(x)\right)}{\mu\left(B_{\rho}(x)\right)} d \mu(x) .
$$

Step 2: refinement of the formula for the integrand. In this step we show that for $\mu$-a.e. $x \in \Omega$

$$
\lim _{\rho \rightarrow 0} \frac{m\left(u, B_{\rho}(x)\right)}{\mu\left(B_{\rho}(x)\right)}=\lim _{\rho \rightarrow 0} \frac{m\left(u_{x}, B_{\rho}(x)\right)}{\mu\left(B_{\rho}(x)\right)}
$$

where $u_{x}$ is given by Proposition 1 (iv).

Let $x \in \Omega$ satisfy

$$
\begin{aligned}
& \lim _{\rho \rightarrow 0} f_{B_{\rho}(x)} a(y) d \mu(y)=a(x)<\infty ; \\
& \lim _{\rho \rightarrow 0} f_{B_{\rho}(x)}\left|\nabla_{\mu} u\right|^{p} d \mu=\left|\nabla_{\mu} u(x)\right|^{p}<\infty ; \\
& \nabla_{\mu} u_{x}(y)=\nabla_{\mu} u(x) \quad \text { for } \mu \text {-a.e. } y \in O ; \\
& \lim _{\rho \rightarrow 0} \frac{1}{\rho}\left(f_{B_{\rho}(x)}\left|u(y)-u_{x}(y)\right|^{p} d \mu(y)\right)^{\frac{1}{p}}=0 .
\end{aligned}
$$

Step 2.1: we prove that $\varlimsup_{\rho \rightarrow 0} \frac{m\left(u_{x}, B_{\rho}(x)\right)}{\mu\left(B_{\rho}(x)\right)} \leqslant \lim _{r \rightarrow 0} \frac{m\left(u, B_{r}(x)\right)}{\mu\left(B_{r}(x)\right)}$ for $\mu$-a.e. $x \in \Omega$. Let $\varepsilon>0$, $\rho>0$ and $t \in] 0,1\left[\right.$. There exists $v \in u+H_{\mu, 0}^{1, p}\left(B_{t \rho}(x) ; \mathbb{R}^{m}\right)$ such that

$$
F\left(v, B_{t \rho}(x)\right) \leqslant \varepsilon \mu\left(B_{t \rho}(x)\right)+m\left(u, B_{t \rho}(x)\right) .
$$


Let $\tau \in] t, 1[$. We consider a Lipschitz function $\varphi: \Omega \rightarrow[0,1]$ which is a Urysohn function for the pair $\left(\Omega \backslash B_{\tau \rho}(x), \bar{B}_{t \rho}(x)\right)$, i.e. satisfying $\varphi \equiv 1$ on $\bar{B}_{t \rho}(x), \varphi \equiv 0$ on $\Omega \backslash B_{\tau \rho}(x)$ and

$$
\left\|D_{\mu} \varphi\right\|_{L_{\mu}^{\infty}} \leqslant \frac{C_{0}}{\rho(\tau-t)}
$$

for some $C_{0}>0$ not depending on $\rho, \tau$ and $t$. We set

$$
w:=\varphi v+(1-\varphi) u_{x} \in u_{x}+H_{\mu, 0}^{1, p}\left(B_{\rho}(x) ; \mathbb{R}^{m}\right)
$$

verifying

$$
w=\left\{\begin{array}{cl}
v & \text { in } B_{t \rho}(x) \\
\varphi u+(1-\varphi) u_{x} & \text { in } B_{\tau \rho}(x) \backslash B_{t \rho}(x) \\
u_{x} & \text { in } B_{\rho}(x) \backslash B_{\tau \rho}(x) .
\end{array}\right.
$$

As in [DM93, pp. 182, Proposition 15.23 and pp. 172, Proposition 14.23] we set

$$
F^{*}(w, B):=\inf \{F(w, O): \mathcal{O}(\Omega) \ni O \supset B\}
$$

for all Borel set $B \subset \Omega$ and all $w \in H_{\mu}^{1, p}\left(\Omega ; \mathbb{R}^{m}\right)$. The functional $F^{*}(w, \cdot)$ is a nonnegative Borel measure which extends to all Borel sets the measure $F(w, \cdot)$, moreover, since the growth condition $\left(\mathrm{C}_{4}\right)$ we have

$$
F^{*}(w, B) \leqslant \int_{B} a(x)+b\left|\nabla_{\mu} w(x)\right|^{p} d \mu(x)
$$

for all Borel set $B \subset \Omega$ and all $w \in H_{\mu}^{1, p}\left(\Omega ; \mathbb{R}^{m}\right)$. Since the locality hypothesis $\left(\mathrm{C}_{2}\right)$ and $(24)$

$$
\begin{aligned}
m\left(u_{x}, B_{\rho}(x)\right) \leqslant F\left(w, B_{\rho}(x)\right) & =F\left(w, B_{t \rho}(x)\right)+F^{*}\left(w, B_{\rho}(x) \backslash B_{t \rho}(x)\right) \\
& \leqslant F\left(v, B_{t \rho}(x)\right)+\int_{B_{\rho}(x) \backslash B_{t \rho}(x)} a(y)+b\left|\nabla_{\mu} w(y)\right|^{p} d \mu(y) .
\end{aligned}
$$

Using the annular decay property (see Definition 4), we have

$$
\begin{aligned}
\int_{B_{\rho}(x) \backslash B_{t \rho}(x)}\left|\nabla_{\mu} w\right|^{p} d \mu & =\int_{B_{\rho}(x) \backslash B_{\tau \rho}(x)}\left|\nabla_{\mu} w\right|^{p} d \mu+\int_{B_{\tau \rho}(x) \backslash B_{t \rho}(x)}\left|\nabla_{\mu} w\right|^{p} d \mu \\
& \leqslant K_{0}(1-\tau)^{\eta} \mu\left(B_{\rho}(x)\right)\left|\nabla_{\mu} u(x)\right|^{p}+\int_{B_{\tau \rho}(x) \backslash B_{t \rho}(x)}\left|\nabla_{\mu} w\right|^{p} d \mu .
\end{aligned}
$$

Since $\nabla_{\mu} w=\varphi \nabla_{\mu} v+(1-\varphi) \nabla_{\mu} u(x)+D_{\mu} \varphi \otimes\left(v-u_{x}\right) \mu$-a.e. in $\Omega$, for some $C_{p}>1$ depending on $p$ only, we have

$$
\begin{aligned}
\int_{B_{\tau \rho}(x) \backslash B_{t \rho}(x)}\left|\nabla_{\mu} w\right|^{p} d \mu \leqslant & C_{p} \int_{B_{\tau \rho}(x) \backslash B_{t \rho}(x)}\left|\nabla_{\mu} u\right|^{p} d \mu+C_{p} \mu\left(B_{\tau \rho}(x) \backslash B_{t \rho}(x)\right)\left|\nabla_{\mu} u(x)\right|^{p} \\
& +C_{p} \int_{B_{\tau \rho}(x) \backslash B_{t \rho}(x)}|| D_{\mu} \varphi \|_{L_{\mu}^{\infty}}^{p}\left|u-u_{x}\right|^{p} d \mu \\
\leqslant & C_{p} \int_{B_{\rho}(x) \backslash B_{t \rho}(x)}\left|\nabla_{\mu} u\right|^{p} d \mu+C_{p} K_{0}(1-t)^{\eta} \mu\left(B_{\rho}(x)\right)\left|\nabla_{\mu} u(x)\right|^{p} \\
& +\frac{C_{p} C_{0}^{p}}{(\tau-t)^{p}} \frac{1}{\rho^{p}} \int_{B_{\rho}(x)}\left|u-u_{x}\right|^{p} d \mu .
\end{aligned}
$$


Collecting (26) and 27) and dividing by $\mu\left(B_{\rho}(x)\right)$, we have

$$
\begin{aligned}
& \frac{1}{\mu\left(B_{\rho}(x)\right)} \int_{B_{\rho}(x) \backslash B_{t \rho}(x)}\left|\nabla_{\mu} w\right|^{p} d \mu \\
\leqslant & K_{0}(1-\tau)^{\eta}\left|\nabla_{\mu} u(x)\right|^{p}+\frac{C_{p}}{\mu\left(B_{\rho}(x)\right)} \int_{B_{\rho}(x) \backslash B_{t \rho}(x)}\left|\nabla_{\mu} u\right|^{p} d \mu+C_{p} K_{0}(1-t)^{\eta}\left|\nabla_{\mu} u(x)\right|^{p} \\
& +\frac{C_{p} C_{0}^{p}}{(\tau-t)^{p}} \frac{1}{\rho^{p}} f_{B_{\rho}(x)}\left|u-u_{x}\right|^{p} d \mu .
\end{aligned}
$$

Passing to the limit $\rho \rightarrow 0$, by taking (21), (19) and Lemma 2 (12) into account, we obtain

$$
\begin{aligned}
\varlimsup_{\rho \rightarrow 0} \frac{1}{\mu\left(B_{\rho}(x)\right)} \int_{B_{\rho}(x) \backslash B_{t \rho}(x)}\left|\nabla_{\mu} w\right|^{p} d \mu & \leqslant K_{0}(1-\tau)^{\eta}\left|\nabla_{\mu} u(x)\right|^{p}+2 C_{p} K_{0}(1-t)^{\eta}\left|\nabla_{\mu} u(x)\right|^{p} \\
& \leqslant 4 C_{p} K_{0}(1-t)^{\eta}\left|\nabla_{\mu} u(x)\right|^{p} .
\end{aligned}
$$

Now, dividing by $\mu\left(B_{\rho}(x)\right)$ the inequality (25) and using (22)

$$
\begin{aligned}
\frac{m\left(u_{x}, B_{\rho}(x)\right)}{\mu\left(B_{\rho}(x)\right)} \leqslant & \frac{F\left(v, B_{t \rho}(x)\right)}{\mu\left(B_{t \rho}(x)\right)}+\frac{1}{\mu\left(B_{\rho}(x)\right)} \int_{B_{\rho}(x) \backslash B_{t \rho}(x)} a+b\left|\nabla_{\mu} w\right|^{p} d \mu \\
\leqslant & \varepsilon+\frac{m\left(u, B_{t \rho}(x)\right)}{\mu\left(B_{t \rho}(x)\right)}+\frac{1}{\mu\left(B_{\rho}(x)\right)} \int_{B_{\rho}(x) \backslash B_{t \rho}(x)} a d \mu \\
& +b \frac{1}{\mu\left(B_{\rho}(x)\right)} \int_{B_{\rho}(x) \backslash B_{t \rho}(x)}\left|\nabla_{\mu} w\right|^{p} d \mu,
\end{aligned}
$$

letting $\rho \rightarrow 0$, by using Lemma 2 (12) together with (18) and (28), we have

$$
\varlimsup_{\rho \rightarrow 0} \frac{m\left(u_{x}, B_{\rho}(x)\right)}{\mu\left(B_{\rho}(x)\right)} \leqslant \varepsilon+\varlimsup_{\rho \rightarrow 0} \frac{m\left(u, B_{t \rho}(x)\right)}{\mu\left(B_{t \rho}(x)\right)}+K_{0}(1-t)^{\eta} a(x)+4 C_{p} K_{0}(1-t)^{\eta}\left|\nabla_{\mu} u(x)\right|^{p} .
$$

Since (18), (19) and the growth condition $\left(\mathrm{C}_{4}\right)$ we have

$$
\varlimsup_{\rho \rightarrow 0} \frac{m\left(u, B_{t \rho}(x)\right)}{\mu\left(B_{t \rho}(x)\right)}=\lim _{r \rightarrow 0} \frac{m\left(u, B_{r}(x)\right)}{\mu\left(B_{r}(x)\right)}<\infty .
$$

Letting $t \rightarrow 1$ in (29) we obtain

$$
\varlimsup_{\rho \rightarrow 0} \frac{m\left(u_{x}, B_{\rho}(x)\right)}{\mu\left(B_{\rho}(x)\right)} \leqslant \varepsilon+\lim _{r \rightarrow 0} \frac{m\left(u, B_{r}(x)\right)}{\mu\left(B_{r}(x)\right)} .
$$

Step 2.2: we prove that $\underline{\lim }_{\rho \rightarrow 0} \frac{m\left(u_{x}, B_{\rho}(x)\right)}{\mu\left(B_{\rho}(x)\right)} \geqslant \lim _{r \rightarrow 0} \frac{m\left(u, B_{r}(x)\right)}{\mu\left(B_{r}(x)\right)}$ for $\mu$-a.e. $x \in \Omega$. Let $\varepsilon>0$, $\rho>0$ and $t \in] 1,2\left[\right.$. There exists $v \in u_{x}+H_{\mu, 0}^{1, p}\left(B_{\rho}(x) ; \mathbb{R}^{m}\right)$ such that

$$
F\left(v, B_{\rho}(x)\right) \leqslant \varepsilon \mu\left(B_{\rho}(x)\right)+m\left(u_{x}, B_{\rho}(x)\right) .
$$

Let $\tau \in] 1, t[$. We consider a Lipschitz function $\varphi: \Omega \rightarrow[0,1]$ which is a Urysohn function for the pair $\left(\Omega \backslash B_{\tau \rho}(x), \bar{B}_{\rho}(x)\right)$, i.e. satisfying $\varphi \equiv 1$ on $\bar{B}_{\rho}(x), \varphi \equiv 0$ on $\Omega \backslash B_{\tau \rho}(x)$ and

$$
\left\|D_{\mu} \varphi\right\|_{L_{\mu}^{\infty}} \leqslant \frac{C_{0}}{\rho(t-\tau)}
$$

for some $C_{0}>0$ not depending on $\rho, \tau$ and $t$. We set $w:=\varphi v+(1-\varphi) u \in u+$ $H_{\mu, 0}^{1, p}\left(B_{\rho}(x) ; \mathbb{R}^{m}\right)$ given by

$$
w=\left\{\begin{array}{cl}
v & \text { in } B_{\rho}(x) \\
\varphi u_{x}+(1-\varphi) u & \text { in } B_{\tau \rho}(x) \backslash B_{\rho}(x) \\
u & \text { in } B_{t \rho}(x) \backslash B_{\tau \rho}(x)
\end{array}\right.
$$


Since the locality hypothesis $\left(\mathrm{C}_{2}\right)$ and $(24)$

$$
\begin{aligned}
m\left(u, B_{t \rho}(x)\right) \leqslant F\left(w, B_{t \rho}(x)\right) & =F\left(w, B_{\rho}(x)\right)+F^{*}\left(w, B_{t \rho}(x) \backslash B_{\rho}(x)\right) \\
& \leqslant F\left(v, B_{t \rho}(x)\right)+\int_{B_{t \rho}(x) \backslash B_{\rho}(x)} a+b\left|\nabla_{\mu} w\right|^{p} d \mu .
\end{aligned}
$$

Using the annular decay property, we have for some $C_{p}>1$ depending on $p$ only

$$
\begin{aligned}
\int_{B_{t \rho}(x) \backslash B_{\rho}(x)}\left|\nabla_{\mu} w\right|^{p} d \mu= & \int_{B_{t \rho}(x) \backslash B_{\tau \rho}(x)}\left|\nabla_{\mu} w\right|^{p} d \mu+\int_{B_{\tau \rho}(x) \backslash B_{\rho}(x)}\left|\nabla_{\mu} w\right|^{p} d \mu \\
\leqslant & C_{p} \int_{B_{t \rho}(x) \backslash B_{\rho}(x)}\left|\nabla_{\mu} u\right|^{p} d \mu+C_{p} K_{0}\left(1-\frac{1}{\tau}\right)^{\eta} \mu\left(B_{\tau \rho}(x)\right)\left|\nabla_{\mu} u(x)\right|^{p} \\
& +\frac{C_{p} C_{0}^{p} t^{p}}{(t-\tau)^{p}} \frac{1}{(t \rho)^{p}} \int_{B_{t \rho}(x)}\left|u-u_{x}\right|^{p} d \mu
\end{aligned}
$$

dividing by $\mu\left(B_{t \rho}(x)\right)$ and passing to the limit $\rho \rightarrow 0$, by taking (21), (19) and (12) into account, we have

$$
\varlimsup_{\rho \rightarrow 0} \frac{1}{\mu\left(B_{t \rho}(x)\right)} \int_{B_{t \rho}(x) \backslash B_{\rho}(x)}\left|\nabla_{\mu} w\right|^{p} d \mu \leqslant 2 C_{p} K_{0}\left(1-\frac{1}{t}\right)^{\eta}\left|\nabla_{\mu} u(x)\right|^{p} .
$$

Now, dividing by $\mu\left(B_{t \rho}(x)\right)$ the inequality (31) and using (30)

$$
\begin{aligned}
\frac{m\left(u, B_{t \rho}(x)\right)}{\mu\left(B_{t \rho}(x)\right)} \leqslant & \varepsilon+\frac{m\left(u_{x}, B_{\rho}(x)\right)}{\mu\left(B_{\rho}(x)\right)}+\frac{1}{\mu\left(B_{t \rho}(x)\right)} \int_{B_{t \rho}(x) \backslash B_{\rho}(x)} a d \mu \\
& +b \frac{1}{\mu\left(B_{t \rho}(x)\right)} \int_{B_{t \rho}(x) \backslash B_{\rho}(x)}\left|\nabla_{\mu} w\right|^{p} d \mu
\end{aligned}
$$

letting $\rho \rightarrow 0$, by using Lemma 2 (12) together with (18) and (33), we have

$$
\varliminf_{\rho \rightarrow 0} \frac{m\left(u, B_{t \rho}(x)\right)}{\mu\left(B_{t \rho}(x)\right)} \leqslant \varepsilon+\varliminf_{\rho \rightarrow 0} \frac{m\left(u_{x}, B_{\rho}(x)\right)}{\mu\left(B_{\rho}(x)\right)}+K_{0}\left(1-\frac{1}{t}\right)^{\eta}\left(a(x)+2 C_{p}\left|\nabla_{\mu} u(x)\right|^{p}\right) .
$$

Since (18), 19) and the growth condition $\left(\mathrm{C}_{4}\right)$ we have

$$
\varliminf_{\rho \rightarrow 0} \frac{m\left(u, B_{t \rho}(x)\right)}{\mu\left(B_{t \rho}(x)\right)}=\lim _{r \rightarrow 0} \frac{m\left(u, B_{r}(x)\right)}{\mu\left(B_{r}(x)\right)}<\infty .
$$

Letting $t \rightarrow 1$ in 34 we obtain

$$
\lim _{r \rightarrow 0} \frac{m\left(u, B_{r}(x)\right)}{\mu\left(B_{r}(x)\right)} \leqslant \varepsilon+\varliminf_{\rho \rightarrow 0} \frac{m\left(u_{x}, B_{\rho}(x)\right)}{\mu\left(B_{\rho}(x)\right)} .
$$

Step 3: proof of (i) and (ii), From Steps 1 and 2, we have

$$
F(u, O)=\int_{O} \lim _{\rho \rightarrow 0} \frac{m\left(u_{x}, B_{\rho}(x)\right)}{\mu\left(B_{\rho}(x)\right)} d \mu(x)
$$

for all $(u, O) \in H_{\mu}^{1, p}\left(\Omega ; \mathbb{R}^{m}\right) \times \mathcal{O}(\Omega)$. Using the extension (23) of $F(u, \cdot)$ to all Borel sets, we have

$$
F^{*}(u, B)=\int_{B} \lim _{\rho \rightarrow 0} \frac{m\left(u_{x}, B_{\rho}(x)\right)}{\mu\left(B_{\rho}(x)\right)} d \mu(x)
$$


for all $(u, B) \in H_{\mu}^{1, p}\left(\Omega ; \mathbb{R}^{m}\right) \times \mathcal{B}(\Omega)$. By Theorem 3, we have $X=\cup_{k=0}^{\infty} X_{k} \cup N$ with $\mu(N)=0$. For each $k \in \mathbb{N}$, consider a nonincreasing sequence of open sets $\left\{O_{k}^{l}\right\}_{l \in \mathbb{N}^{*} \subset}$ $\mathcal{O}(\Omega)$ satisfying $O_{k}^{l} \supset\left(X_{k} \cap \Omega\right)$ and $\mu\left(O_{k}^{l} \backslash\left(X_{k} \cap \Omega\right)\right) \leqslant \frac{1}{l}$ for all $l \in \mathbb{N}^{*}$, and

$$
B_{k}:=\bigcap_{l=1}^{\infty} O_{k}^{l} \supset\left(X_{k} \cap \Omega\right) \text { with } \mu\left(B_{k}\right)=\mu\left(X_{k} \cap \Omega\right) \text { for all } k \in \mathbb{N} .
$$

Then we can write $\Omega=\cup_{k=0}^{\infty} B_{k} \cup N_{0}$ with $N_{0}:=N \cup \cup_{k=0}^{\infty} B_{k} \backslash\left(X_{k} \cap \Omega\right)$. Since $F^{*}(u, \cdot)$ is a Borel measure absolutely continuous with respect to $\mu$, by using $\left(\mathrm{C}_{3}\right)$ and the monotone convergence theorem, we have

$$
\begin{aligned}
F(u, O)=F^{*}(u, O) & =\sum_{k=0}^{\infty} F^{*}\left(u, O \cap B_{k}\right) \\
& =\sum_{k=0}^{\infty} \int_{O \cap B_{k}} \varlimsup_{\rho \rightarrow 0} \frac{m\left(u_{x}, B_{\rho}(x)\right)}{\mu\left(B_{\rho}(x)\right)} d \mu(x) \\
& =\int_{O} \sum_{k=0}^{\infty} \varlimsup_{\rho \rightarrow 0} \inf _{\varphi \in H_{\mu, 0}^{1, p}\left(B_{\rho}(x) ; \mathbb{R}^{m}\right)} \frac{F\left(u_{x}+\varphi, B_{\rho}(x)\right)}{\mu\left(B_{\rho}(x)\right)} \mathbb{1}_{B_{k}}(x) d \mu(x) \\
& =\int_{O} \sum_{k=0}^{\infty} \varlimsup_{\rho \rightarrow 0} \frac{m\left(\nabla_{\mu} u(x) \cdot \gamma^{k}, B_{\rho}(x)\right)}{\mu\left(B_{\rho}(x)\right)} \mathbb{1}_{B_{k}}(x) d \mu(x)
\end{aligned}
$$

for all $(u, O) \in H_{\mu}^{1, p}\left(\Omega ; \mathbb{R}^{m}\right) \times \mathcal{O}(\Omega)$. We set

$$
f(x, \xi):=\sum_{k=0}^{\infty} f^{k}(x, \xi) \text { where } f^{k}(x, \xi):=\varlimsup_{\rho \rightarrow 0} \frac{m\left(\xi \cdot \gamma^{k}, B_{\rho}(x)\right)}{\mu\left(B_{\rho}(x)\right)} \mathbb{1}_{B_{k}}(x)
$$

for all $(x, \xi) \in \Omega \times \mathbb{M}$ and all $k \in \mathbb{N}$. First, (ii) holds because for every $k \in \mathbb{N}$, for $\mu$-a.e. $x \in X_{k} \cap \Omega$ and for every $\xi \in \mathbb{M}$

$$
f(x, \xi):=\varlimsup_{\rho \rightarrow 0} \frac{m\left(\xi \cdot \gamma^{k}, B_{\rho}(x)\right)}{\mu\left(B_{\rho}(x)\right)}
$$

since $B_{k}=\left(X_{k} \cap \Omega\right) \cup N_{k}$ with $\mu\left(N_{k}\right)=0$. Second, if we set

$$
f_{\rho}^{k}(x, \xi):=\frac{m\left(\xi \cdot \gamma^{k}, B_{\rho}(x)\right)}{\mu\left(B_{\rho}(x)\right)} \mathbb{1}_{B_{k}}(x)
$$

then $f^{k}(x, \xi)=\varlimsup_{\rho \rightarrow 0} f_{\rho}^{k}(x, \xi)$ and $f_{\rho}^{k}(x, \xi)=\inf _{l \geqslant 1} f_{\rho}^{k, l}(x, \xi)$ where

$$
f_{\rho}^{k, l}(x, \xi):=\frac{m\left(\xi \cdot \gamma^{k}, B_{\rho}(x)\right)}{\mu\left(B_{\rho}(x)\right)} \mathbb{1}_{O_{k}^{l}}(x)
$$

for all $(x, \xi) \in \Omega \times \mathbb{M}$. Now, to prove (i), we need to show that $f$ is Borel measurable, it suffices to prove that each $f_{\rho}^{k, l}$ is Borel measurable, indeed, this would imply that each $f_{\rho}^{k}$ is Borel measurable as infimum on countable set of $f_{\rho}^{k, l}$, then the upper limit defining $f^{k}$ can be computed as $\rho \rightarrow 0$ along a suitable countable set, and finally $f$ is Borel measurable as a countable sum of Borel measurable functions. In fact, the following lemma shows that each $f_{\rho}^{k, l}$ is lower semicontinuous.

Lemma 4. Let $k \in \mathbb{N}, \rho>0$ and $l \in \mathbb{N}^{*}$. Let $(\bar{x}, \bar{\xi}) \in \Omega \times \mathbb{M}$ and two sequences $\left\{x_{n}\right\}_{n \in \mathbb{N}} \subset \Omega$, $\left\{\xi_{n}\right\}_{n \in \mathbb{N}} \subset \mathbb{M}$ satisfy $\lim _{n \rightarrow \infty} d\left(x_{n}, \bar{x}\right)=0$ and $\lim _{n \rightarrow \infty}\left|\xi_{n}-\bar{\xi}\right|=0$. Then

$$
\varliminf_{n \rightarrow \infty} f_{\rho}^{k, l}\left(x_{n}, \xi_{n}\right) \geqslant f_{\rho}^{k, l}(\bar{x}, \bar{\xi}) \text {. }
$$


Proof of Lemma 4. Fix $k \in \mathbb{N}$ and $\rho>0$. Assume that there exists a subsequence (not relabelled) $\left\{\xi_{n}\right\}_{n \in \mathbb{N}} \subset \mathbb{M}$ such that

$$
\infty>\varliminf_{n \rightarrow \infty} m\left(\xi_{n} \cdot \gamma^{k}, B_{\rho}(\bar{x})\right)=\lim _{n \rightarrow \infty} m\left(\xi_{n} \cdot \gamma^{k}, B_{\rho}(\bar{x})\right) .
$$

Note that by the growth condition (the right inequality in) $\left(\mathrm{C}_{4}\right)$

$$
\sup _{n \in \mathbb{N}} m\left(\xi_{n} \cdot \gamma^{k}, B_{\rho}(\bar{x})\right) \leqslant \sup _{n \in \mathbb{N}} \int_{B_{\rho}(\bar{x})}\left(a(x)+b\left|\xi_{n}\right|^{p}\right) d \mu(x)<\infty .
$$

There exists $\left\{\varphi_{n}\right\}_{n \in \mathbb{N}^{*}} \subset H_{\mu, 0}^{1, p}\left(B_{\rho}(\bar{x}) ; \mathbb{R}^{m}\right)$ such that for all $n \geqslant 1$

$$
\frac{1}{n}+m\left(\xi_{n} \cdot \gamma^{k}, B_{\rho}(\bar{x})\right) \geqslant F\left(\xi_{n} \cdot \gamma^{k}+\varphi_{n}, B_{\rho}(\bar{x})\right) .
$$

By the coercivity condition (the left inequality in) $\left(\mathrm{C}_{4}\right)$ and $(36)$ we get

$$
\sup _{n \in \mathbb{N}^{*}} \int_{B_{\rho}(\bar{x})}\left|\nabla_{\mu} \varphi_{n}\right|^{p} d \mu<\infty
$$

Using the $p$-Sobolev inequality Proposition 1 (iii), we see that $\left\{\varphi_{n}\right\}_{n \in \mathbb{N}^{*}} \subset H_{\mu, 0}^{1, p}\left(B_{\rho}(\bar{x}) ; \mathbb{R}^{m}\right)$ is bounded, so, by Theorem 4, there exists a subsequence which converges strongly in $L_{\mu}^{p}\left(B_{\rho}(\bar{x}) ; \mathbb{R}^{m}\right)$ and weakly in $H_{\mu, 0}^{1, p}\left(B_{\rho}(\bar{x}) ; \mathbb{R}^{m}\right)$ to $\bar{\varphi}$. By the lower semicontinuity hypothesis $\left(\mathrm{C}_{5}\right)$, we have

$$
\varliminf_{n \rightarrow \infty} F\left(\xi_{n} \cdot \gamma^{k}+\varphi_{n}, B_{\rho}(\bar{x})\right) \geqslant F\left(\bar{\xi} \cdot \gamma^{k}+\bar{\varphi}, B_{\rho}(\bar{x})\right) \geqslant m\left(\bar{\xi} \cdot \gamma^{k}, B_{\rho}(\bar{x})\right),
$$

thus, passing to the limit $n \rightarrow \infty$ in (37), we obtain

$$
\varliminf_{n \rightarrow \infty} m\left(\xi_{n} \cdot \gamma^{k}, B_{\rho}(\bar{x})\right) \geqslant m\left(\bar{\xi} \cdot \gamma^{k}, B_{\rho}(\bar{x})\right) .
$$

Fix $l \in \mathbb{N}^{*}$. We can assume that $\bar{x} \in O_{k}^{l}$, otherwise $f_{\rho}^{k, l}(\bar{x}, \cdot)=0$. Thus, there exists $N \in \mathbb{N}$ such that $x_{n} \in O_{k}^{l}$ for all $n \geqslant N$.

We claim that

$$
\varliminf_{n \rightarrow \infty} m\left(\xi_{n} \cdot \gamma^{k}, B_{\rho}\left(x_{n}\right)\right)-m\left(\xi_{n} \cdot \gamma^{k}, B_{\rho}(\bar{x})\right) \geqslant 0
$$

Indeed, fix $n \geqslant N$, there exists a sequence $\left\{\varphi_{n}^{m}\right\}_{m \in \mathbb{N}^{*}} \subset H_{\mu, 0}^{1, p}\left(B_{\rho}\left(x_{n}\right) ; \mathbb{R}^{m}\right)$ such that for all $m \geqslant 1$

$\infty>\int_{B_{\rho}(\bar{x})} a(x)+b\left|\xi_{n}\right|^{p} d \mu(x) \geqslant m\left(\xi_{n} \cdot \gamma^{k}, B_{\rho}\left(x_{n}\right)\right) \geqslant-\frac{1}{m}+F\left(\xi_{n} \cdot \gamma^{k}+\varphi_{n}^{m}, B_{\rho}\left(x_{n}\right)\right)$,

using the coercivity condition $\left(\mathrm{C}_{4}\right)$, we have

$$
\sup _{m \in \mathbb{N}^{*}} \int_{B_{\rho}\left(x_{n}\right)}\left|\nabla_{\mu} \varphi_{n}^{m}\right|^{p} d \mu<\infty .
$$

We apply the $p$-Sobolev inequality Proposition 1(iii), we see that the sequence $\left\{\varphi_{n}^{m}\right\}_{m \in \mathbb{N}^{*}} \subset$ $H_{\mu, 0}^{1, p}\left(B_{\rho}\left(x_{n}\right) ; \mathbb{R}^{m}\right)$ is bounded. So, by Theorem 4 , there exists a subsequence (not relabelled) which converges strongly in $L_{\mu}^{p}\left(B_{\rho}\left(x_{n}\right) ; \mathbb{R}^{m}\right)$ and weakly in $H_{\mu, 0}^{1, p}\left(B_{\rho}\left(x_{n}\right) ; \mathbb{R}^{m}\right)$ to 
$\varphi_{n}^{\infty}$ as $m \rightarrow \infty$. By the lower semicontinuity hypothesis $\left(\mathrm{C}_{5}\right)$, we have

$$
\begin{aligned}
m\left(\xi_{n} \cdot \gamma^{k}, B_{\rho}\left(x_{n}\right)\right) \geqslant & \underset{m \rightarrow \infty}{\lim _{m}} F\left(\xi_{n} \cdot \gamma^{k}+\varphi_{n}^{m}, B_{\rho}\left(x_{n}\right)\right) \\
\geqslant & F\left(\xi_{n} \cdot \gamma^{k}+\varphi_{n}^{\infty}, B_{\rho}\left(x_{n}\right)\right) \\
= & F\left(\xi_{n} \cdot \gamma^{k}+\varphi_{n}^{\infty}, B_{\rho}\left(x_{n}\right)\right)-F\left(\xi_{n} \cdot \gamma^{k}+\varphi_{n}^{\infty}, B_{\rho}(\bar{x})\right) \\
& +F\left(\xi_{n} \cdot \gamma^{k}+\varphi_{n}^{\infty}, B_{\rho}(\bar{x})\right) \\
\geqslant & F\left(\xi_{n} \cdot \gamma^{k}+\varphi_{n}^{\infty}, B_{\rho}\left(x_{n}\right)\right)-F\left(\xi_{n} \cdot \gamma^{k}+\varphi_{n}^{\infty}, B_{\rho}(\bar{x})\right) \\
& +m\left(\xi_{n} \cdot \gamma^{k}, B_{\rho}(\bar{x})\right)
\end{aligned}
$$

For every $n \geqslant N$ and every open set $O \subset \Omega$, we have by using $\left(\mathrm{C}_{4}\right)$

$$
F\left(\xi_{n} \cdot \gamma^{k}, O\right) \leqslant \int_{O}\left(a+b \sup _{n \in \mathbb{N}}\left|\xi_{n}\right|^{p}\right) d \mu=\nu(O),
$$

we also have for every Borel set $B \subset \Omega$

$$
F^{*}\left(\xi_{n} \cdot \gamma^{k}, B\right) \leqslant \nu(B)
$$

where $F^{*}$ is given by (23) and $\nu:=\left.\left(a+b \sup _{n \in \mathbb{N}}\left|\xi_{n}\right|^{p}\right) \mu\right|_{\Omega}$. By $\left(\mathrm{C}_{1}\right)$, we have

$$
\begin{aligned}
& F\left(\xi_{n} \cdot \gamma^{k}+\varphi_{n}^{\infty}, B_{\rho}\left(x_{n}\right)\right)-F\left(\xi_{n} \cdot \gamma^{k}+\varphi_{n}^{\infty}, B_{\rho}(\bar{x})\right) \\
& =F^{*}\left(\xi_{n} \cdot \gamma^{k}+\varphi_{n}^{\infty}, B_{\rho}\left(x_{n}\right) \backslash B_{\rho}(\bar{x})\right)-F^{*}\left(\xi_{n} \cdot \gamma^{k}+\varphi_{n}^{\infty}, B_{\rho}(\bar{x}) \backslash B_{\rho}\left(x_{n}\right)\right) \\
& =F^{*}\left(\xi_{n} \cdot \gamma^{k}+\varphi_{n}^{\infty}, B_{\rho}\left(x_{n}\right) \backslash B_{\rho}(\bar{x})\right)-F^{*}\left(\xi_{n} \cdot \gamma^{k}, B_{\rho}(\bar{x}) \backslash B_{\rho}\left(x_{n}\right)\right) \\
& \geqslant-F^{*}\left(\xi_{n} \cdot \gamma^{k}, B_{\rho}(\bar{x}) \backslash B_{\rho}\left(x_{n}\right)\right) \\
& \geqslant-\nu\left(B_{\rho}(\bar{x}) \backslash B_{\rho}\left(x_{n}\right)\right) .
\end{aligned}
$$

Since $\lim _{n \rightarrow \infty} d\left(x_{n}, \bar{x}\right)=0$, we have $\lim _{n \rightarrow \infty} \nu\left(B_{\rho}(\bar{x}) \backslash B_{\rho}\left(x_{n}\right)\right)=0$, so (39) holds. Now, using (38) and (39), we obtain

$$
\begin{aligned}
& \underline{\lim _{n \rightarrow \infty} m}\left(\xi_{n} \cdot \gamma^{k}, B_{\rho}\left(x_{n}\right)\right) \\
& =\underline{\lim }_{n \rightarrow \infty}\left(m\left(\xi_{n} \cdot \gamma^{k}, B_{\rho}\left(x_{n}\right)\right)-m\left(\xi_{n} \cdot \gamma^{k}, B_{\rho}(\bar{x})\right)+m\left(\xi_{n} \cdot \gamma^{k}, B_{\rho}(\bar{x})\right)\right) \\
& \geqslant \underline{\varliminf_{n \rightarrow \infty}}\left(m\left(\xi_{n} \cdot \gamma^{k}, B_{\rho}\left(x_{n}\right)\right)-m\left(\xi_{n} \cdot \gamma^{k}, B_{\rho}(\bar{x})\right)\right)+\underline{\lim }_{n \rightarrow \infty} m\left(\xi_{n} \cdot \gamma^{k}, B_{\rho}(\bar{x})\right) \\
& \geqslant m\left(\bar{\xi} \cdot \gamma^{k}, B_{\rho}(\bar{x})\right) .
\end{aligned}
$$

Fix $\varepsilon>0$. Since the measure of the boundary of balls is zero (see Remark 2), we have $\lim _{n \rightarrow \infty} \mu\left(B_{\rho}\left(x_{n}\right)\right)=\mu\left(B_{\rho}(\bar{x})\right)$, so, for some integer $N^{\prime} \geqslant N$ and for all $n \geqslant N^{\prime}$

$$
\mu\left(B_{\rho}\left(x_{n}\right)\right) \leqslant \varepsilon+\mu\left(B_{\rho}(\bar{x})\right) .
$$

It follows that for all $n \geqslant N^{\prime}$

$$
f_{\rho}^{k, l}\left(x_{n}, \xi_{n}\right)=\frac{m\left(\xi_{n} \cdot \gamma^{k}, B_{\rho}\left(x_{n}\right)\right)}{\mu\left(B_{\rho}\left(x_{n}\right)\right)} \geqslant \frac{m\left(\xi_{n} \cdot \gamma^{k}, B_{\rho}\left(x_{n}\right)\right)}{\varepsilon+\mu\left(B_{\rho}(\bar{x})\right)},
$$

letting $n \rightarrow \infty$ and then $\varepsilon \rightarrow 0$ we obtain 35 . 
Step 4: proof of (iv) and (v), By $\left(\mathrm{C}_{4}\right)$ and the integral representation obtained in the previous step, we have for every $(u, O) \in H_{\mu}^{1, p}\left(\Omega ; \mathbb{R}^{m}\right) \times \mathcal{O}(\Omega)$

$$
c \int_{O}\left|\nabla_{\mu} u(x)\right|^{p} d \mu(x) \leqslant \int_{O} f\left(x, \nabla_{\mu} u(x)\right) d \mu(x) \leqslant \int_{O} a(x)+b\left|\nabla_{\mu} u(x)\right|^{p} d \mu(x)
$$

where $c>0, b \geqslant 0$ and $a \in L_{\mu}^{1}(\Omega)$ are given by $\left(\mathrm{C}_{4}\right)$. By the Lebesgue differentiation theorem, we deduce that for every $u \in H_{\mu}^{1, p}\left(\Omega ; \mathbb{R}^{m}\right)$ and for $\mu$-a.e. $x \in \Omega$

$$
g_{1}\left(x, \nabla_{\mu} u(x)\right) \leqslant f\left(x, \nabla_{\mu} u(x)\right) \leqslant g_{2}\left(x, \nabla_{\mu} u(x)\right)
$$

where for every $(x, \xi) \in \Omega \times \mathbb{M}$

$$
g_{1}(x, \xi)=c|\xi|^{p} \quad \text { and } \quad g_{2}(x, \xi)=a(x)+b|\xi|^{p},
$$

Using the Borel measurable function $N$ of the Lemma 1 , we set for every $(x, \xi) \in \Omega \times \mathbb{M}$

$$
\widetilde{g}_{1}(x, \xi):=c N(x, \xi)^{p} \quad \text { and } \quad \widetilde{g}_{2}(x, \xi):=\widetilde{a}+b N(x, \xi)^{p}
$$

where $\widetilde{a}$ is a Borel measurable function satisfying $a=\widetilde{a} \mu$-a.e. in $\Omega$ (see [HKST15, Proposition 3.3.23, pp. 59 and Lemma 3.3.28, pp. 62]). We have for $\mu$-a.e. $x \in \Omega$ and for every $\xi \in \mathbb{M}$

$$
\widetilde{g}_{1}(x, \xi)=g_{1}(x, \xi) \quad \text { and } \quad \tilde{g}_{2}(x, \xi)=g_{2}(x, \xi),
$$

thus, using (40), we can write for every $u \in H_{\mu}^{1, p}\left(\Omega ; \mathbb{R}^{m}\right)$ and for $\mu$-a.e. $x \in \Omega$

$$
\widetilde{g}_{1}\left(x, \nabla_{\mu} u(x)\right) \leqslant f\left(x, \nabla_{\mu} u(x)\right) \leqslant \widetilde{g}_{2}\left(x, \nabla_{\mu} u(x)\right) .
$$

We can apply Lemma 9 to obtain (iv), To see(v), we apply Corollary 2 .

\section{Proof of Theorem 2}

4.1. The De Giorgi-Letta lemma. Let $X=(X, d)$ be a metric space, let $\mathcal{O}(X)$ be the class of open subsets of $X$ and let $\mathcal{B}(X)$ be the class of Borel subsets of $X$, i.e. the smallest $\sigma$ algebra containing the open (or equivalently the closed) subsets of $X$. The following result is due to De Giorgi and Letta (see [DGL77] and also [But89, Lemma 3.3.6 p. 105]).

Lemma 5. Let $\mathcal{S}: \mathcal{O}(X) \rightarrow[0, \infty]$ be an increasing set function, i.e. $\mathcal{S}(U) \leqslant \mathcal{S}(V)$ for all $U, V \in \mathcal{O}(X)$ such $U \subset V$, satisfying the following four conditions:

(i) $\mathcal{S}(\varnothing)=0$;

(ii) $\mathcal{S}$ is superadditive, i.e. $\mathcal{S}(U \cup V) \geqslant \mathcal{S}(U)+\mathcal{S}(V)$ for all $U, V \in \mathcal{O}(X)$ such that $U \cap V=$ $\varnothing$

(iii) $\mathcal{S}$ is subadditive, i.e. $\mathcal{S}(U \cup V) \leqslant \mathcal{S}(U)+\mathcal{S}(V)$ for all $U, V \in \mathcal{O}(X)$;

(iv) there exists a finite regular measure $\alpha: \mathcal{B}(X) \rightarrow[0, \infty]$ such that $\mathcal{S}(U) \leqslant \alpha(U)$ for all $U \in \mathcal{O}(X)$.

Then, $\mathcal{S}$ can be uniquely extended to a finite regular measure $\mathcal{S}^{*}: \mathcal{B}(X) \rightarrow[0, \infty]$ defined by

$$
\mathcal{S}^{*}(B)=\inf \{\mathcal{S}(O): \mathcal{O}(X) \ni O \supset B\},
$$

and which satisfies $\mathcal{S}^{*}(B) \leqslant \alpha(B)$ for all $B \in \mathcal{B}(X)$. 
4.2. Proof of Theorem 2, We need the following lemma:

Lemma 6. Let $u \in H_{\mu}^{1, p}\left(\Omega ; \mathbb{R}^{m}\right)$ and $O \in \mathcal{O}(\Omega)$. Then

(i) the functional $\bar{F}(u, \cdot)$ is a measure;

(ii) $c \int_{O}\left|\nabla_{\mu} u\right|^{p} d \mu \leqslant \bar{F}(u, O) \leqslant \int_{O}\left(a+b\left|\nabla_{\mu} u\right|^{p}\right) d \mu$ where $c>0, a \in L_{\mu}^{1}(\Omega)$ and $b \geqslant 0$ are given by $\left(\mathrm{C}_{4}\right)$ :

(iii) $\bar{m}(u, O)=m(u, O)$ where $\bar{m}(u, O)=\inf \left\{\bar{F}(u+\varphi, O): \varphi \in H_{\mu, 0}^{1, p}\left(X ; \mathbb{R}^{m}\right)\right\}$.

The Lemma (i) and (ii) insures that $\bar{F}$ satisfies $\left(\mathrm{C}_{1}\right)$ and $\left(\mathrm{C}_{4}\right)$. It is direct to see that $\bar{F}$ verifies $\left(\mathrm{C}_{2}\right)$, and we refer to [DM93, Proposition 16.15, pp. 185] for $\left(\mathrm{C}_{3}\right)$, Therefore we can apply Theorem 1 to have the following integral representation: for every $O \in \mathcal{O}(\Omega)$ and every $u \in H_{\mu}^{1, p}\left(\Omega ; \mathbb{R}^{m}\right)$

$$
\bar{F}(u, O)=\int_{O} \bar{f}\left(x, \nabla_{\mu} u(x)\right) d \mu(x),
$$

where $\bar{f}$ is a Borel measurable function satisfying, for every $k \in \mathbb{N}$, for $\mu$-a.e. $x \in \Omega \cap X_{k}$ and for every $\xi \in \mathbb{M}$

$$
\bar{f}(x, \xi):=\varlimsup_{\rho \rightarrow 0} \inf _{\varphi \in H_{\mu, 0}^{1, p}\left(B_{\rho}(x) ; \mathbb{R}^{m}\right)} \frac{\bar{F}\left(\xi \cdot \gamma^{k}(\cdot)+\varphi, B_{\rho}(x)\right)}{\mu\left(B_{\rho}(x)\right)} ;
$$

We obtain the relaxed integrand stated in Theorem 2(ii) by using Lemma 6[(iii), Note that Theorem 2 (iv) is a consequence of Theorem 1/(iv).

Proof of Lemma 6(i), Fix $u \in H_{\mu}^{1, p}\left(\Omega ; \mathbb{R}^{m}\right)$. Using the right inequality in $\left(\mathrm{C}_{4}\right)$ we see that

$$
\bar{F}(u, O) \leqslant \int_{O}\left(a+b\left|\nabla_{\mu} u\right|^{p}\right) d \mu \text { for all } O \in \mathcal{O}(\Omega) .
$$

Thus, the condition(iv) of Lemma 5 is satisfied with $\alpha=\left(a+b\left|\nabla_{\mu} u\right|^{p}\right) \mu$ (which is absolutely continuous with respect to $\mu$ ). On the other hand, it is easily seen that the conditions (i) and (ii) of Lemma 5 are satisfied. The proof will be complete by proving the condition (iii) of Lemma 5 , i.e.

$$
\bar{F}(u, U \cup V) \leqslant \bar{F}(u, U)+\bar{F}(u, V) \text { for all } U, V \in \mathcal{O}(\Omega) .
$$

To show (43) we need the following lemma:

Lemma 7. If $U, V, Z, T \in \mathcal{O}(\Omega)$ are such that $\bar{Z} \subset U$ and $T \subset V$, then

$$
\bar{F}(u, Z \cup T) \leqslant \bar{F}(u, U)+\bar{F}(u, V) .
$$

Proof of Lemma 7. Let $\left\{u_{n}\right\}_{n \in \mathbb{N}}$ and $\left\{v_{n}\right\}_{n \in \mathbb{N}}$ be two sequences in $H_{\mu}^{1, p}\left(\Omega ; \mathbb{R}^{m}\right)$ such that:

$$
\begin{aligned}
& u_{n} \rightarrow u \text { in } L_{\mu}^{p}\left(\Omega ; \mathbb{R}^{m}\right) ; \\
& v_{n} \rightarrow u \text { in } L_{\mu}^{p}\left(\Omega ; \mathbb{R}^{m}\right) ; \\
& \lim _{n \rightarrow \infty} F\left(u_{n}, U\right)=\bar{F}(u, U)<\infty ; \\
& \lim _{n \rightarrow \infty} F\left(v_{n}, V\right)=\bar{F}(u, V)<\infty .
\end{aligned}
$$


Fix $\delta \in] 0, \operatorname{dist}(Z, \partial U)\left[\right.$ with $\partial U:=\bar{U} \backslash U$, fix any integer $q \geqslant 1$ and consider $W_{i}^{-}, W_{i}^{+} \subset \Omega$ given by:

$$
\begin{aligned}
W_{i}^{-} & :=\left\{x \in \Omega: \operatorname{dist}(x, Z) \leqslant \frac{\delta}{3}\left(1+\frac{i-1}{q}\right)\right\} \\
\text { and } W_{i}^{+} & :=\left\{x \in \Omega: \frac{\delta}{3}\left(1+\frac{i}{q}\right) \leqslant \operatorname{dist}(x, Z)\right\},
\end{aligned}
$$

where $i \in\{1, \cdots, q\}$. For every $i \in\{1, \cdots, q\}$ there exists a Uryshon function $\varphi_{i} \in \operatorname{Lip}(\Omega)$ for the pair $\left(W_{i}^{+}, W_{i}^{-}\right)$. Fix $n \in \mathbb{N}$. Define $w_{n}^{i} \in H_{\mu}^{1, p}\left(\Omega ; \mathbb{R}^{m}\right)$ by

$$
w_{n}^{i}:=\varphi_{i} u_{n}+\left(1-\varphi_{i}\right) v_{n} .
$$

Setting $W_{i}:=\Omega \backslash\left(W_{i}^{-} \cup W_{i}^{+}\right)$and using Theorem 3 (iii) and (7) we have

$$
\nabla_{\mu} w_{n}^{i}= \begin{cases}\nabla_{\mu} u_{n} & \text { in } W_{i}^{-} \\ D_{\mu} \varphi_{i} \otimes\left(u_{n}-v_{n}\right)+\varphi_{i} \nabla_{\mu} u_{n}+\left(1-\varphi_{i}\right) \nabla_{\mu} v_{n} & \text { in } W_{i} \\ \nabla_{\mu} v_{n} & \text { in } W_{i}^{+} .\end{cases}
$$

Noticing that $Z \cup T=\left((Z \cup T) \cap W_{i}^{-}\right) \cup\left(W \cap W_{i}\right) \cup\left(T \cap W_{i}^{+}\right)$with $(Z \cup T) \cap W_{i}^{-} \subset U$, $T \cap W_{i}^{+} \subset V$ and $W:=T \cap\left\{x \in U: \frac{\delta}{3}<\operatorname{dist}(x, Z)<\frac{2 \delta}{3}\right\}$ we deduce that

$$
F\left(w_{n}^{i}, Z \cup T\right) \leqslant F\left(u_{n}, U\right)+F\left(v_{n}, V\right)+F\left(w_{n}^{i}, W \cap W_{i}\right)
$$

for all $i \in\{1, \ldots, q\}$. Moreover, from the right inequality in $\left(\mathrm{C}_{4}\right)$ we see that for each $i \in\{1, \ldots, q\}$,

$$
\begin{aligned}
F\left(w_{n}^{i}, W \cap W_{i}\right) \leqslant & b D_{\mu} \varphi_{i}\left\|_{L_{\mu}^{\infty}\left(\Omega ; \mathbb{R}^{N}\right)}^{p}\right\| u_{n}-v_{n} \|_{L_{\mu}^{p}\left(\Omega ; \mathbb{R}^{m}\right)}^{p} \\
& +\int_{W \cap W_{i}}\left(a+b\left|\nabla_{\mu} u_{n}\right|^{p}+b\left|\nabla_{\mu} v_{n}\right|^{p}\right) d \mu
\end{aligned}
$$

with $c:=2^{2 p} \beta$. Substituting (52) into (51) and averaging these inequalities, it follows that for every $n \in \mathbb{N}$ and every $q \geqslant 1$, there exists $i_{n, q} \in\{1, \ldots, q\}$ such that

$$
\begin{aligned}
F\left(w_{n}^{i_{n, q}}, Z \cup T\right) \leqslant & F\left(u_{n}, U\right)+F\left(v_{n}, V\right)+\frac{b}{q} \sum_{i=1}^{q}\left\|D_{\mu} \varphi_{i}\right\|_{L_{\mu}^{\infty}\left(\Omega ; \mathbb{R}^{N}\right)}^{p}\left\|u_{n}-v_{n}\right\|_{L_{\mu}^{p}\left(\Omega ; \mathbb{R}^{m}\right)}^{p} \\
& +\frac{1}{q}\left(\int_{V} a d \mu+b \int_{U}\left|\nabla_{\mu} u_{n}\right|^{p} d \mu+b \int_{V}\left|\nabla_{\mu} v_{n}\right|^{p} d \mu\right) .
\end{aligned}
$$

On the other hand, by (45) and (46) we have:

$$
\lim _{n \rightarrow \infty}\left\|u_{n}-v_{n}\right\|_{L_{\mu}^{p}\left(\Omega ; \mathbb{R}^{m}\right)}^{p}=0 \quad \text { and } \quad \lim _{n \rightarrow \infty}\left\|w_{n}^{i_{n, q}}-u\right\|_{L_{\mu}^{p}\left(\Omega ; \mathbb{R}^{m}\right)}^{p}=0 \text { for all } q \geqslant 1 .
$$

Moreover, using (47) and (48) together with the left inequality in $\left(\mathrm{C}_{4}\right)$ we see that:

$$
\varlimsup_{n \rightarrow \infty} \int_{U}\left|\nabla_{\mu} u_{n}(x)\right|^{p} d \mu(x)<\infty \quad \text { and } \quad \varlimsup_{n \rightarrow \infty} \int_{V}\left|\nabla_{\mu} v_{n}(x)\right|^{p} d \mu(x)<\infty .
$$

Letting $t \rightarrow \infty$ (and taking (47) and (48) into account) we deduce that for every $q \geqslant 1$,

$$
\bar{F}(u, Z \cup T) \leqslant \varliminf_{n \rightarrow \infty} F\left(w_{n}^{i_{n, q}}, Z \cup T\right) \leqslant \bar{F}(u, U)+\bar{F}(u, V)+\frac{C}{q}
$$

with $C:=\int_{V} a d \mu+b \varlimsup_{n \rightarrow \infty} \int_{U}\left|\nabla_{\mu} u_{n}\right|^{p} d \mu+b \varlimsup_{n \rightarrow \infty} \int_{V}\left|\nabla_{\mu} v_{n}\right|^{p} d \mu$, and the inequality (44) follows from (53) by letting $q \rightarrow \infty$. 
We now prove (43). Fix $O_{1}, O_{2} \in \mathcal{O}(\Omega)$. Fix $\varepsilon>0$ and consider $C, D \in \mathcal{O}(\Omega)$ such that $\bar{C} \subset O_{1}, \bar{D} \subset O_{2}$ and

$$
\int_{E} a(x)+b\left|\nabla_{\mu} u(x)\right|^{p} d \mu(x)<\varepsilon
$$

with $E:=O_{1} \cup O_{2} \backslash \overline{C \cup D}$. Then $\bar{F}(u, E) \leqslant \varepsilon$ by $(42)$ Let $\widehat{C}, \widehat{D}, \widehat{\widehat{C}}, \widehat{\hat{D}} \in \mathcal{O}(\Omega)$ be such that

$$
\bar{C} \subset \widehat{C} \subset \overline{\widehat{C}} \subset \widehat{\hat{C}} \subset \overline{\hat{C}} \subset O_{1} \quad \text { and } \quad \bar{D} \subset \widehat{D} \subset \overline{\widehat{D}} \subset \widehat{\hat{D}} \subset \overline{\hat{D}} \subset O_{2}
$$

Applying Lemma 7 with $U=\widehat{\hat{C}} \cup \widehat{\hat{D}}, V=T=E$ and $Z=\widehat{C} \cup \widehat{D}$ gives

$$
\bar{F}\left(u, O_{1} \cup O_{2}\right) \leqslant \bar{F}(u, \widehat{\widehat{C}} \cup \widehat{\hat{D}})+\varepsilon,
$$

since $Z \cup T=\left(O_{1} \cup O_{2} \backslash \overline{C \cup D}\right) \cup(\widehat{C} \cup \widehat{D}) \supset\left(O_{1} \cup O_{2} \backslash \overline{C \cup D}\right) \cup(\bar{C} \cup \bar{D})=O_{1} \cup O_{2}$, i.e $Z \cup T=O_{1} \cup O_{2}$. Using again Lemma 7 with $U=O_{1}, V=O_{2}, Z=\widehat{\widehat{C}}$ and $T=\widehat{\hat{D}}$ we obtain

$$
\bar{F}(u, \widehat{\hat{C}} \cup \widehat{\hat{D}}) \leqslant \bar{F}\left(u, O_{1}\right)+\bar{F}\left(u, O_{2}\right)
$$

hence

$$
\bar{F}\left(u, O_{1} \cup O_{2}\right) \leqslant \bar{F}\left(u, O_{1}\right)+\bar{F}\left(u, O_{2}\right)+\varepsilon,
$$

and (43) follows by letting $\varepsilon \rightarrow 0$.

We can extract and isolate the following lemma by examining the proof of Lemma 7 above:

Lemma 8. If $U, V, Z, T \in \mathcal{O}(X)$ are such that $\bar{Z} \subset U$ and $T \subset V$, and if $\left\{u_{n}\right\}_{n \in \mathbb{N}}$ is a sequence in $H_{\mu}^{1, p}\left(\Omega ; \mathbb{R}^{m}\right)$ such that $u_{n} \rightarrow u$ in $L_{\mu}^{p}\left(\Omega ; \mathbb{R}^{m}\right)$, then for every $\left.\delta \in\right] 0$, dist $(Z, \partial U)$ [ there exists a sequence $\left\{w_{n}\right\}_{n \in \mathbb{N}}$ in $H_{\mu}^{1, p}\left(\Omega ; \mathbb{R}^{m}\right)$ such that:

$$
w_{n} \rightarrow u \text { in } L_{\mu}^{p}\left(\Omega ; \mathbb{R}^{m}\right), w_{n}=u \quad \mu \text {-a.e. in }\left\{x \in X: \operatorname{dist}(x, Z) \geqslant \frac{2 \delta}{3}\right\} \text {, }
$$

and for every $n \in \mathbb{N}$ and every $q \geqslant 1$,

$$
\begin{aligned}
F\left(w_{n}, Z \cup T\right) \leqslant & F\left(u_{n}, U\right)+F(u, V)+\frac{b}{q} \sum_{i=1}^{q}\left\|D_{\mu} \varphi_{i}\right\|_{L_{\mu}^{\infty}\left(\Omega ; \mathbb{R}^{N}\right)}^{p}\left\|u_{n}-u\right\|_{L_{\mu}^{p}\left(\Omega ; \mathbb{R}^{m}\right)}^{p} \\
& +\frac{1}{q}\left(\int_{V} a d \mu+b \int_{U}\left|\nabla_{\mu} u_{n}\right|^{p} d \mu+b \int_{V}\left|\nabla_{\mu} u\right|^{p} d \mu\right),
\end{aligned}
$$

where each $\varphi_{i} \in \operatorname{Lip}(X)$ is a Uryshon function for the pair $\left(W_{i}^{+}, W_{i}^{-}\right)$defined in (49) and (50).

Proof of Lemma 6(iii), Let $u \in H_{\mu}^{1, p}\left(\Omega ; \mathbb{R}^{m}\right)$ and $O \in \mathcal{O}(\Omega)$. It is sufficient to show that

$$
\bar{m}(u, O) \geqslant m(u, O),
$$

where $\bar{m}(u, O)=\inf \left\{\bar{F}(u+\varphi, O): \varphi \in H_{\mu, 0}^{1, p}\left(O ; \mathbb{R}^{m}\right)\right\}$.

Fix $\varepsilon>0$. There exists $\varphi \in H_{\mu, 0}^{1, p}\left(O ; \mathbb{R}^{m}\right)$ such that

$$
\varepsilon+\bar{m}(u, O) \geqslant \bar{F}(u+\varphi, O) .
$$

There exists $\left\{\varphi_{n}\right\}_{n \in \mathbb{N}}$ such that $\varphi_{n} \rightarrow \varphi$ in $L_{\mu}^{p}\left(\Omega ; \mathbb{R}^{m}\right)$ and

$$
\infty>\varepsilon+\bar{m}(u, O) \geqslant \bar{F}(u+\varphi, O)=\lim _{n \rightarrow \infty} F\left(u+\varphi_{n}, O\right) \text {. }
$$


Apply Lemma 8 with

$$
\begin{aligned}
U & :=O, \quad Z:=\left\{x \in O: \operatorname{dist}(x, \Omega \backslash O)>\frac{2 \delta}{3}\right\}, \\
T & =V:=V_{\delta}=\{x \in O: \operatorname{dist}(x, \Omega \backslash O)<\delta\} \\
\text { and } u_{n} & :=u+\varphi_{n} .
\end{aligned}
$$

There exists a sequence $\left\{w_{n}\right\}_{n \in \mathbb{N}}$ in $H_{\mu}^{1, p}\left(\Omega ; \mathbb{R}^{m}\right)$ such that:

$$
w_{n} \rightarrow u \text { in } L_{\mu}^{p}\left(\Omega ; \mathbb{R}^{m}\right) \text { and } w_{n}=u+\varphi=u \mu \text {-a.e. in } \Omega \backslash O,
$$

and for every $n \in \mathbb{N}$ and every $q \geqslant 1$,

$$
\begin{aligned}
m(u, O) \leqslant F\left(w_{n}, O\right) \leqslant & F\left(u+\varphi_{n}, O\right)+F\left(u+\varphi, V_{\delta}\right) \\
& +\frac{b}{q} \sum_{i=1}^{q}\left\|D_{\mu} \varphi_{i}\right\|_{L_{\mu}^{\infty}\left(\Omega ; \mathbb{R}^{N}\right)}^{p}\left\|\varphi_{n}-\varphi\right\|_{L_{\mu}^{p}\left(\Omega ; \mathbb{R}^{m}\right)}^{p} \\
& +\frac{1}{q}\left(\int_{V} a d \mu+b \int_{U}\left|\nabla_{\mu} u+\nabla_{\mu} \varphi_{n}\right|^{p} d \mu+b \int_{V}\left|\nabla_{\mu} u+\nabla_{\mu} \varphi\right|^{p} d \mu\right),
\end{aligned}
$$

since $O=Z \cup T$. Moreover, using (55) together with the left inequality in $\left(\mathrm{C}_{4}\right)$ we see that:

$$
\varlimsup_{n \rightarrow \infty} \int_{O}\left|\nabla_{\mu} u+\nabla_{\mu} \varphi_{n}\right|^{p} d \mu<\infty .
$$

Letting $n \rightarrow \infty$ we deduce that for every $q \geqslant 1$,

$$
m(u, O) \leqslant \varepsilon+\bar{m}(u, O)+\int_{V_{\delta}} a+b\left|\nabla_{\mu} u+\nabla_{\mu} \varphi\right|^{p} d \mu+\frac{C}{q}
$$

with $C:=\int_{V} a d \mu+b \varlimsup_{n \rightarrow \infty} \int_{U}\left|\nabla_{\mu} u+\nabla_{\mu} \varphi_{n}\right|^{p} d \mu+b \int_{V}\left|\nabla_{\mu} u+\nabla_{\mu} \varphi\right|^{p} d \mu$, and (54) follows from (56) by letting $q \rightarrow \infty, \delta \rightarrow 0$ and $\varepsilon \rightarrow 0$.

Proof of Lemma 6(ii), Fix $u \in H_{\mu}^{1, p}\left(\Omega ; \mathbb{R}^{m}\right)$. Using the right inequality in $\left(\mathrm{C}_{4}\right)$ we see that

$$
\bar{F}(u, O) \leqslant \int_{O}\left(a+b\left|\nabla_{\mu} u\right|^{p}\right) d \mu \text { for all } O \in \mathcal{O}(\Omega) .
$$

On the other side by the lower semicontinuity of the norm, we have

$$
\begin{aligned}
\bar{F}(u, O) & \geqslant c \inf \left\{\varliminf_{n \rightarrow \infty} \int_{O}\left|\nabla_{\mu} u_{n}(x)\right|^{p} d \mu(x): u_{n} \rightarrow u \text { in } L_{\mu}^{p}\left(\Omega ; \mathbb{R}^{m}\right)\right\} \\
& =c \int_{O}\left|\nabla_{\mu} u(x)\right|^{p} d \mu(x) .
\end{aligned}
$$

\section{Proof of Corollary 1}

Define $F: H_{\mu}^{1, p}\left(\Omega ; \mathbb{R}^{m}\right) \times \mathcal{O}(\Omega) \rightarrow[0, \infty]$ by

$$
F(u, O):=\int_{O} f\left(x, \nabla_{\mu} u(x)\right) d \mu(x) .
$$

We see that $F$ satisfies $\left(\mathrm{C}_{1}\right),\left(\mathrm{C}_{4}\right)$. We apply Theorem 2 , we have for every $O \in \mathcal{O}(\Omega)$ and every $u \in H_{\mu}^{1, p}\left(\Omega ; \mathbb{R}^{m}\right)$

$$
\bar{F}(u, O)=\int_{O} \bar{f}\left(x, \nabla_{\mu} u(x)\right) d \mu(x)
$$


where the Borel integrand $\bar{f}$ is given, for $\mu$-a.e. $x \in \Omega$ and for every $\xi \in \mathbb{M}$, by

$$
\bar{f}(x, \xi):=\varlimsup_{\rho \rightarrow 0} \inf _{\varphi \in H_{\mu, 0}^{1, p}\left(B_{\rho}(x) ; \mathbb{R}^{m}\right)} f_{B_{\rho}(x)} f\left(y, \xi+\nabla_{\mu} \varphi\right) d \mu .
$$

Assume that (ii) holds, i.e. $F(u, O)=\bar{F}(u, O)$ for all $(u, O) \in H_{\mu}^{1, p}\left(\Omega ; \mathbb{R}^{m}\right) \times \mathcal{O}(\Omega)$. Using the Lebesgue differentiation theorem, we have for every $u \in H_{\mu}^{1, p}\left(\Omega ; \mathbb{R}^{m}\right)$ and for $\mu$-a.e. $x \in \Omega$

$$
\bar{f}\left(x, \nabla_{\mu} u(x)\right)=f\left(x, \nabla_{\mu} u(x)\right) .
$$

It follows, from Corollary 2 below, that for $\mu$-a.e. $x \in \Omega$ and for every $\xi \in \mathbb{M}$

$$
\bar{f}(x, \xi)=f(x, \xi) .
$$

Now, assume that (i) holds, i.e. we have the equality (57). Using Theorem 2 we have for all $(u, O) \in H_{\mu}^{1, p}\left(\Omega ; \mathbb{R}^{m}\right) \times \mathcal{O}(\Omega)$

$$
\bar{F}(u, O)=\int_{O} \bar{f}\left(x, \nabla_{\mu} u(x)\right) d \mu(x)=\int_{O} f\left(x, \nabla_{\mu} u(x)\right) d \mu(x)=F(u, O)
$$

which means that $F(\cdot, O)$ is $L_{\mu}^{p}$-lower semicontinuous.

\section{Auxiliary Results}

6.1. Integrands and Lusin-type theorems. The Lemma 9 below is a version of an Alberti result [Alb91, Corollary 6, pp. 112] concerning inequalities of Borel integrands, in the setting of metric measure spaces. In fact, using an extension to metric measure spaces of the Alberti result on Lusin-type for gradients by David [Dav15], we are able to rewrite the proof given by Alberti in this framework with minor changes.

Lemma 9. Let $U \subset X$ be an open subset with $\mu(U)<\infty$, and let $h, g: U \times \mathbb{M} \rightarrow \overline{\mathbb{R}}$ be two Borel functions such that for every $u \in \operatorname{Lip}_{0}\left(U ; \mathbb{R}^{m}\right)$ and for $\mu$-a.e. $x \in U$

$$
h\left(x, \nabla_{\mu} u(x)\right) \leqslant g\left(x, \nabla_{\mu} u(x)\right) .
$$

Then for $\mu$-a.e. $x \in U$ and for every $\xi \in \mathbb{M}$

$$
h(x, \xi) \leqslant g(x, \xi) .
$$

For the proof, we adapt slightly the one of Alberti [Alb91] to our setting. It consists in showing that $\mu(\pi(B))=0$ where $\pi(B)$ is the projection onto $U$ of the set $B$ of all $(x, \xi) \in U \times \mathbb{M}$ which do not satisfy (59). For this, a generalized version of the measurable selection theorem allows to find a measurable selection not satisfying $(59)$ at every point of $\pi(B)$, then using the Lusin-type theorem for gradient of David [Dav15], we will be able to say, because of (58), that $\mu(\pi(B))$ is as small as we wish.

Proof of Lemma 9. Depending on the needs of the proof, we state only partially the result of Lusin-type theorems for gradients in metric measure spaces:

Proposition 2. [Dav15, Theorem 1.3, pp. 297] For every open subset $U \subset X$ with $\mu(U)<\infty$, every collection of Borel measurable functions $\left\{w_{k}: X_{k} \cap U \rightarrow \mathbb{M}\right\}_{k \in \mathbb{N}}$ and every $\varepsilon>0$, there exist an open set $O \subset U$ and a Lipschitz function $u \in \operatorname{Lip}_{0}\left(U ; \mathbb{R}^{m}\right)$ such that

$$
\mu(O) \leqslant \varepsilon \mu(U) \text { and } w_{k}=\nabla_{\mu} u \quad \mu \text {-a.e. in } X_{k} \cap(U \backslash O) \quad \text { for all } k \in \mathbb{N} \text {. }
$$

Define the Borel set $B:=\{(x, \xi) \in U \times \mathbb{M}: h(x, \xi)>g(x, \xi)\}$. Suppose $B \neq \varnothing$, otherwise there is nothing to prove. Consider $\pi(B) \subset U$ the projection onto $U$ of $B$. The projection theorem [CV77, Theorem III.23, pp. 75] shows that $\pi(B)$ is $\mu$-measurable, i.e. $\pi(B) \in \mathcal{B}_{\mu}(X)$. The rest of the proof is dedicated to showing that $\mu(\pi(B))=0$. 
Let $\Lambda_{B}: \pi(B) \rightrightarrows \mathbb{M}$ be the multifunction defined by

$$
\Lambda_{B}(x):=\{\xi \in \mathbb{M}:(x, \xi) \in B\} .
$$

Note that $\Lambda_{B}(x) \neq \varnothing$ for all $x \in \pi(B)$ since $\pi(B) \neq \varnothing$. Moreover, its graph is the Borel set $B$, so, we can apply the Aumann-Sainte Beuve theorem [CV77, Theorem III.22, pp. 74] (see [SB74, Theorem 3, pp. 119]), which gives a $\left(\mathcal{B}_{\mu}(X), \mathcal{B}(\mathrm{M})\right)$-measurable function (a measurable selection) $\sigma: \pi(B) \rightarrow \mathbb{M}$ satisfying

$$
\sigma(x) \in \Lambda_{B}(x) \text { for all } x \in \pi(B) .
$$

Define $\tilde{\sigma}: U \rightarrow \mathbb{M}$ by $\widetilde{\sigma}:=\sigma \mathbb{1}_{\pi(B)}$. From [HKST15, Proposition 3.3.23, pp. 59 and Lemma 3.3.28, pp. 62], there exists a Borel function $w: U \rightarrow \mathbb{M}$ and $\mathcal{N} \in \mathcal{B}_{\mu}(X)$ with $\mu(\mathcal{N})=0$ such that $w=\widetilde{\sigma}$ on $U \backslash \mathcal{N}$. Fix $\varepsilon>0$. Apply Proposition 2 with $w_{k}:=w\left\lfloor_{X_{k}}\right.$. There exist an open set $O \subset U$, a Lipschitz function $u \in \operatorname{Lip}_{0}\left(U ; \mathbb{R}^{m}\right)$ and $\tilde{\mathcal{N}} \in \mathcal{B}_{\mu}(X)$ with $\mu(\tilde{\mathcal{N}})=0$ such that

$$
\mu(O) \leqslant \varepsilon \mu(U) \text { and } w=\nabla_{\mu} u \quad(X \backslash \tilde{\mathcal{N}}) \cap(U \backslash O) .
$$

For every $x \in(X \backslash \tilde{\mathcal{N}}) \cap(U \backslash O) \cap \pi(B) \backslash \mathcal{N}$ we have $\sigma(x)=\nabla_{\mu} u(x)$, according to 60 we can write

$$
h\left(x, \nabla_{\mu} u(x)\right)>g\left(x, \nabla_{\mu} u(x)\right) .
$$

From (58), there exists $\mathcal{N}_{0} \in \mathcal{B}_{\mu}(X)$ with $\mu\left(\mathcal{N}_{0}\right)=0$ such that for every $x \in U \backslash \mathcal{N}_{0}$

$$
h\left(x, \nabla_{\mu} u(x)\right) \leqslant g\left(x, \nabla_{\mu} u(x)\right) .
$$

It follows that $U \backslash \mathcal{N}_{0} \cap(X \backslash \tilde{\mathcal{N}}) \cap(U \backslash O) \cap \pi(B) \backslash \mathcal{N}=\varnothing$, i.e. $\pi(B) \subset O \cup \mathcal{N} \cup \mathcal{N}_{0} \cup \tilde{\mathcal{N}}$, then $\mu(\pi(B)) \leqslant \mu(O) \leqslant \varepsilon \mu(U)$. Letting $\varepsilon \rightarrow 0$, we get $\mu(\pi(B))=0$.

Corollary 2. Let $U \subset X$ be an open subset with $\mu(U)<\infty$ and let $h, g: U \times \mathbb{M} \rightarrow \overline{\mathbb{R}}$ be two Borel functions such that for every $u \in \operatorname{Lip}_{0}\left(U ; \mathbb{R}^{m}\right)$ and for $\mu$-a.e. $x \in U$

$$
h\left(x, \nabla_{\mu} u(x)\right)=g\left(x, \nabla_{\mu} u(x)\right) .
$$

Then for $\mu$-a.e. $x \in U$ and every $\xi \in \mathbb{M}$ it holds

$$
h(x, \xi)=g(x, \xi) .
$$

6.2. $\boldsymbol{H}_{\mu}^{1, p}$-quasiconvex integrands. Here we deduce easily Theorem 1 (iii) and Theorem 2(iii) from Proposition 3 below.

Definition 5. Let $f: X \times \mathbb{M} \longrightarrow[0, \infty]$ be a Borel measurable function. Let $A$ be a measurable subset of $X$. We say that $f$ is $H_{\mu}^{1, p}$-quasiconvex on $A$ at $\xi \in \mathbb{M}$ if for $\mu$-a.e. $x \in A$ it holds

$$
f(x, \xi) \leqslant \underline{\lim _{\rho \rightarrow 0}} \inf _{\varphi \in H_{\mu, 0}^{1, p}\left(B_{\rho}(x) ; \mathbb{R}^{m}\right)} f_{\bar{B}_{\rho}(x)} f\left(y, \xi+\nabla_{\mu} \varphi\right) d \mu .
$$

When $f$ is $H_{\mu}^{1, p}$-quasiconvex on $A$ at every $\xi \in \mathbb{M}$ we say that $f$ is $H_{\mu}^{1, p}$-quasiconvex on $A$, and if $A=X$ we say that $f$ is $H_{\mu}^{1, p}$-quasiconvex.

Remark 3. As a direct consequence of the Lebesgue differentiation theorem and the definition of $H_{\mu}^{1, p}$-quasiconvexity, an integrand $f: X \times \mathbb{M} \longrightarrow[0, \infty]$ is $H_{\mu}^{1, p}$-quasiconvex at $\xi \in \mathbb{M}$ satisfying $f(\cdot, \xi) \in L_{\mu, \text { loc }}^{1}(X)$ if and only if for $\mu$-a.e. $x \in X$

$$
f(x, \xi)=\lim _{\rho \rightarrow 0} \inf _{\varphi \in H_{\mu, 0}^{1, p}\left(B_{\rho}(x) ; \mathbb{R}^{m}\right)} f_{\bar{B}_{\rho}(x)} f\left(y, \xi+\nabla_{\mu} \varphi\right) d \mu .
$$


In [AHM20, Theorem 3, pp. 10] we proved:

Proposition 3. Let $f: X \times \mathbb{M} \longrightarrow[0, \infty]$ be a p-coercive Borel measurable integrand, i.e. satisfying for some $c>0$, for $\mu$-a.e. $x \in X$ and for every $\xi \in \mathbb{M}$

$$
f(x, \xi) \geqslant c|\xi|^{p} \text {. }
$$

Assume that for every $u,\left\{u_{\varepsilon}\right\}_{\varepsilon>0} \subset H_{\mu}^{1, p}\left(X ; \mathbb{R}^{m}\right)$ satisfying $\lim _{\varepsilon \rightarrow 0}\left\|u_{\varepsilon}-u\right\|_{L_{\mu}^{p}\left(X ; \mathbb{R}^{m}\right)}=0$, it holds

$$
\varliminf_{\varepsilon \rightarrow 0} \int_{O} f\left(x, \nabla_{\mu} u_{\varepsilon}\right) d \mu \geqslant \int_{O} f\left(x, \nabla_{\mu} u\right) d \mu
$$

for all open set $O \in \mathcal{O}(X)$ with $\mu(O)<\infty$.

Then $f$ is $H_{\mu}^{1, p}$-quasiconvex on $X$ at every $\xi \in \mathbb{M}$ satisfying $f(\cdot, \xi) \in L_{\mu, \text { loc }}^{1}(X)$.

6.3. Integral representation of the Vitali envelope of a set function. This part is devoted to the integral representation of the Vitali envelope of a set function defined on open subsets of $X$, it is partly inspired by [BB00, BFM98, DMM86]. Then we apply it to the set function $m(u, \cdot)$.

For each open set $O \subset \Omega$, we denote by $\mathfrak{B}(O) \subset \mathcal{O}(O)$ the class of all open balls $B$ of $O$.

6.3.1. Vitali envelopes of set functions. Let $G: \mathfrak{B}(\Omega) \rightarrow \mathbb{R}$ be a set function. We define the lower Vitali envelope of $G$ with respect to $\mu$

$$
\mathcal{O}(\Omega) \ni O \longmapsto G_{-}^{*}(O):=\sup _{\varepsilon>0} \inf \left\{\sum_{i \in I} G\left(B_{i}\right):\left\{B_{i}\right\}_{i \in I} \in \mathcal{V}^{\varepsilon}(O)\right\}
$$

and the upper Vitali envelope with respect to $\mu$

$$
\mathcal{O}(\Omega) \ni O \longmapsto G_{+}^{*}(O):=\inf _{\varepsilon>0} \sup \left\{\sum_{i \in I} G\left(B_{i}\right):\left\{B_{i}\right\}_{i \in I} \in \mathcal{V}^{\varepsilon}(O)\right\},
$$

where for every $\varepsilon>0$

$$
\begin{aligned}
& \mathcal{V}^{\varepsilon}(O):=\left\{\left\{B_{i}\right\}_{i \in I} \subset \mathfrak{B}(O): I\right. \text { is countable, } \mu\left(O \backslash \cup_{i \in I} B_{i}\right)=0, \bar{B}_{i} \subset O, \\
&\left.0<\operatorname{diam}\left(B_{i}\right) \leqslant \varepsilon \text { and } \bar{B}_{i} \cap \bar{B}_{j}=\varnothing \text { for all } i \neq j\right\} .
\end{aligned}
$$

Remark 4. If $G$ is the trace on $\mathfrak{B}(\Omega)$ of a positive Borel measure $\lambda$ on $\Omega$ which is absolutely continuous with respect to $\mu$ then $G_{ \pm}^{*}(O)=\lambda(O)$ for all $O \in \mathcal{O}(\Omega)$.

Let $G: \mathfrak{B}(\Omega) \rightarrow \mathbb{R}$ be a set function. Define the lower and the upper derivatives at $x \in \Omega$ of $G$ with respect to $\mu$ as follows

$$
\begin{aligned}
& d_{\mu}^{-} G(x):=\lim _{\rho \rightarrow 0} \inf \left\{\frac{G(B)}{\mu(B)}: x \in B \in \mathfrak{B}(\Omega), 0<\operatorname{diam}(B) \leqslant \rho\right\} \\
& d_{\mu}^{+} G(x):=\lim _{\rho \rightarrow 0} \sup \left\{\frac{G(B)}{\mu(B)}: x \in B \in \mathfrak{B}(\Omega), 0<\operatorname{diam}(B) \leqslant \rho\right\} .
\end{aligned}
$$

We say that $G$ is $\mu$-differentiable in $O \in \mathcal{O}(\Omega)$ if for $\mu$-a.e. $x \in O$ it holds

$$
-\infty<d_{\mu}^{-} G(x)=d_{\mu}^{+} G(x)<\infty,
$$

in this case, we denote the common value by $d_{\mu} G(x)$ and

$$
d_{\mu} G(x)=\lim _{\rho \rightarrow 0} \frac{G\left(B_{\rho}(x)\right)}{\mu\left(B_{\rho}(x)\right)} .
$$


Under domination and subadditivity conditions (see conditions (i) and (ii) below), a set function defined on open sets is $\mu$-differentiable, and the lower and upper Vitali envelopes are equal and admit an integral representation with density its derivative, for the proof see [AHM18, Theorem 3.17, pp. 65]:

Theorem 5. Let $G: \mathcal{O}(\Omega) \rightarrow \mathbb{R}$ satisfy:

(i) there exists a positive Radon measure $\alpha$ on $\Omega$ absolutely continuous with respect to $\mu$, i.e. $\alpha \ll \mu$, satisfying

$$
|G(O)| \leqslant \alpha(O) \quad \text { for all } O \in \mathcal{O}(\Omega) ;
$$

(ii) for every $U, V, O \in \mathcal{O}(\Omega)$ with $U \cap V=\varnothing, U \subset O, V \subset O$ and $\mu(O \backslash(U \cup V))=0$ it holds

$$
G(O) \leqslant G(U)+G(V) .
$$

Then $G$ is $\mu$-differentiable with $\Omega \ni x \longmapsto \lim _{\rho \rightarrow 0} \frac{G\left(B_{\rho}(x)\right)}{\mu\left(B_{\rho}(x)\right)} \in L_{\mu}^{1}(\Omega)$ and for every $O \in \mathcal{O}(\Omega)$

$$
G_{+}^{*}(O)=G_{-}^{*}(O)=\int_{O} \lim _{\rho \rightarrow 0} \frac{G\left(B_{\rho}(x)\right)}{\mu\left(B_{\rho}(x)\right)} d \mu(x) .
$$

Lemma 10. Assume that $\left(\mathrm{C}_{1}\right)$ and $\left(\mathrm{C}_{4}\right)$ hold. Then

(i) for every $u \in H_{\mu}^{1, p}\left(\Omega ; \mathbb{R}^{m}\right)$ the set function $m(u, \cdot)$ is $\mu$-differentiable and

$$
\Omega \ni x \longmapsto \lim _{\rho \rightarrow 0} \frac{m\left(u, B_{\rho}(x)\right)}{\mu\left(B_{\rho}(x)\right)} \in L_{\mu}^{1}(\Omega) ;
$$

(ii) for every $O \in \mathcal{O}(\Omega)$ and every $u \in H_{\mu}^{1, p}\left(\Omega ; \mathbb{R}^{m}\right)$ we have

$$
m_{-}^{*}(u, O)=\int_{O} \lim _{\rho \rightarrow 0} \frac{m\left(u, B_{\rho}(x)\right)}{\mu\left(B_{\rho}(x)\right)} d \mu(x) .
$$

Proof of Lemma 10, Let $u \in H_{\mu}^{1, p}\left(\Omega ; \mathbb{R}^{m}\right)$. The proof consists in verifying the hypothesis of Theorem 5 for the set function $G(\cdot)=m(u, \cdot)$.

Let us show that the set function $m(u, \cdot): \mathcal{O}(\Omega) \rightarrow \mathbb{R}_{+}$is subadditive. Let $\left(U_{1}, U_{2}, W\right) \in$ $\mathcal{O}(\Omega)^{3}$ be three open sets such that $U_{1} \subset W, U_{2} \subset W$ with $\mu\left(W \backslash\left(U_{1} \cup U_{2}\right)\right)=0$ and $U_{1} \cap U_{2}=\varnothing$. We can assume that $m\left(u, U_{1}\right)<\infty$ and $m\left(u, U_{2}\right)<\infty$. There exists $v_{i} \in u+H_{\mu, 0}^{1, p}\left(U_{i} ; \mathbb{R}^{m}\right)$ such that

$$
\varepsilon+m\left(u, U_{i}\right) \geqslant F\left(v_{i}, U_{i}\right) .
$$

Set $v_{0}:=\sum_{i=1}^{2} v_{i} \mathbb{1}_{U_{i}}+u \mathbb{1}_{\Omega \backslash\left(U_{1} \cup U_{2}\right)} \in u+H_{\mu, 0}^{1, p}\left(W ; \mathbb{R}^{m}\right)$. We have, by using $\left(\mathrm{C}_{1}\right)$ and $\left(\mathrm{C}_{4}\right)$.

$$
\begin{aligned}
F\left(v_{0}, W\right)=F^{*}\left(v_{0}, W\right) & =F\left(v_{0}, U_{1} \cup U_{2}\right)+F^{*}\left(v_{0}, W \backslash\left(U_{1} \cup U_{2}\right)\right) \\
& \leqslant \sum_{i=1}^{2} F\left(v_{0}, U_{i}\right)+\int_{W \backslash\left(U_{1} \cup U_{2}\right)} a(x)+b\left|\nabla_{\mu} v_{0}\right|^{p} d \mu \\
& =\sum_{i=1}^{2} F\left(v_{0}, U_{i}\right) .
\end{aligned}
$$

From (65), it follows that

$$
m(u, W) \leqslant F\left(v_{0}, W\right) \leqslant \sum_{i=1}^{2} F\left(v_{0}, U_{i}\right) \leqslant 2 \varepsilon+\sum_{i=1}^{2} m\left(u, U_{i}\right),
$$

Theorem 5 (ii) holds by letting $\varepsilon \rightarrow 0$. 
Now, if we set

$$
\alpha(O):=\int_{O} a(x)+b\left|\nabla_{\mu} u(x)\right|^{p} d \mu(x) \quad \text { for all open set } O \subset \Omega
$$

then $\alpha$ is a positive Radon measure (in fact $\mu$ is a Radon measure, see [HKST15, Proposition 3.3.44, pp. 81]) on $\Omega$ absolutely continuous with respect to $\mu$, and

$$
m(u, O) \leqslant F(u, O) \leqslant \alpha(O) \quad \text { for all open set } O \subset \Omega
$$

since $\left(\mathrm{C}_{4}\right)$, i.e. Theorem 5 (i) holds.

\section{REFERENCES}

[AHM15] Omar Anza Hafsa and Jean-Philippe Mandallena. On the relaxation of variational integrals in metric Sobolev spaces. Adv. Calc. Var., 8(1):69-91, 2015.

[AHM17] Omar Anza Hafsa and Jean-Philippe Mandallena. $\Gamma$-convergence of nonconvex integrals in Cheeger-Sobolev spaces and homogenization. Adv. Calc. Var., 10(4):381-405, 2017.

[AHM18] Omar Anza Hafsa and Jean-Philippe Mandallena. Relaxation of nonconvex unbounded integrals with general growth conditions in Cheeger-Sobolev spaces. Bull. Sci. Math., 142:49-93, 2018.

[AHM20] Omar Anza Hafsa and Jean-Philippe Mandallena. Lower semicontinuity of integrals of the calculus of variations in Cheeger-Sobolev spaces. Calc. Var. Partial Differential Equations, 59(2):Paper No. 53, 32, 2020.

[Alb91] Giovanni Alberti. A Lusin type theorem for gradients. J. Funct. Anal., 100(1):110-118, 1991.

[BB00] Guy Bouchitté and Michel Bellieud. Regularization of a set function-application to integral representation. Ricerche Mat., 49(suppl.):79-93, 2000. Contributions in honor of the memory of Ennio De Giorgi (Italian).

[BB11] Anders Björn and Jana Björn. Nonlinear potential theory on metric spaces, volume 17 of EMS Tracts in Mathematics. European Mathematical Society (EMS), Zürich, 2011.

[BBS97] Guy Bouchitte, Giuseppe Buttazzo, and Pierre Seppecher. Energies with respect to a measure and applications to low-dimensional structures. Calc. Var. Partial Differential Equations, 5(1):3754, 1997.

[BDM85] Giuseppe Buttazzo and Gianni Dal Maso. Integral representation and relaxation of local functionals. Nonlinear Anal., 9(6):515-532, 1985.

[BFLM02] Guy Bouchitté, Irene Fonseca, Giovanni Leoni, and Luísa Mascarenhas. A global method for relaxation in $W^{1, p}$ and in $\mathrm{SBV}_{p}$. Arch. Ration. Mech. Anal., 165(3):187-242, 2002.

[BFM98] Guy Bouchitté, Irene Fonseca, and Luisa Mascarenhas. A global method for relaxation. Arch. Rational Mech. Anal., 145(1):51-98, 1998.

[Bjö00] Jana Björn. $L^{q}$-differentials for weighted Sobolev spaces. Michigan Math. J., 47(1):151-161, 2000.

[BM84] J. M. Ball and F. Murat. $W^{1, p}$-quasiconvexity and variational problems for multiple integrals. $J$. Funct. Anal., 58(3):225-253, 1984.

[Buc99] Stephen M. Buckley. Is the maximal function of a Lipschitz function continuous? Ann. Acad. Sci. Fenn. Math., 24(2):519-528, 1999.

[But89] Giuseppe Buttazzo. Semicontinuity, relaxation and integral representation in the calculus of variations, volume 207 of Pitman Research Notes in Mathematics Series. Longman Scientific \& Technical, Harlow, 1989.

[Che99] J. Cheeger. Differentiability of Lipschitz functions on metric measure spaces. Geom. Funct. Anal., 9(3):428-517, 1999.

[CJLP02] Gregory A. Chechkin, Vasili V. Jikov, Dag Lukkassen, and Andrey L. Piatnitski. On homogenization of networks and junctions. Asymptot. Anal., 30(1):61-80, 2002.

[CM98] Tobias H. Colding and William P. Minicozzi, II. Liouville theorems for harmonic sections and applications. Comm. Pure Appl. Math., 51(2):113-138, 1998.

[CPS07] G. A. Chechkin, A. L. Piatnitski, and A. S. Shamaev. Homogenization, volume 234 of Translations of Mathematical Monographs. American Mathematical Society, Providence, RI, 2007. Methods and applications, Translated from the 2007 Russian original by Tamara Rozhkovskaya.

[CV77] C. Castaing and M. Valadier. Convex analysis and measurable multifunctions. Lecture Notes in Mathematics, Vol. 580. Springer-Verlag, Berlin-New York, 1977. 
[Dav15] Guy C. David. Lusin-type theorems for Cheeger derivatives on metric measure spaces. Anal. Geom. Metr. Spaces, 3(1):296-312, 2015.

[DGL77] E. De Giorgi and G. Letta. Une notion générale de convergence faible pour des fonctions croissantes d'ensemble. Ann. Scuola Norm. Sup. Pisa Cl. Sci. (4), 4(1):61-99, 1977.

[DM93] Gianni Dal Maso. An introduction to $\Gamma$-convergence. Progress in Nonlinear Differential Equations and their Applications, 8. Birkhäuser Boston Inc., Boston, MA, 1993.

[DMM86] Gianni Dal Maso and Luciano Modica. Integral functionals determined by their minima. Rend. Sem. Mat. Univ. Padova, 76:255-267, 1986.

[FHK99] B. Franchi, P. Hajłasz, and P. Koskela. Definitions of Sobolev classes on metric spaces. Ann. Inst. Fourier (Grenoble), 49(6):1903-1924, 1999.

[GH13] Jasun Gong and Piotr Hajłasz. Differentiability of $p$-harmonic functions on metric measure spaces. Potential Anal., 38(1):79-93, 2013.

[GT01] Vladimir Gol'dshtein and Marc Troyanov. Axiomatic theory of Sobolev spaces. Expo. Math., 19(4):289-336, 2001.

[Haj03] Piotr Hajłasz. Sobolev spaces on metric-measure spaces. In Heat kernels and analysis on manifolds, graphs, and metric spaces (Paris, 2002), volume 338 of Contemp. Math., pages 173-218. Amer. Math. Soc., Providence, RI, 2003.

[Hei07] Juha Heinonen. Nonsmooth calculus. Bull. Amer. Math. Soc. (N.S.), 44(2):163-232, 2007.

[HK98] Juha Heinonen and Pekka Koskela. Quasiconformal maps in metric spaces with controlled geometry. Acta Math., 181(1):1-61, 1998.

[HK00] Piotr Hajłasz and Pekka Koskela. Sobolev met Poincaré. Mem. Amer. Math. Soc., 145(688):x+101, 2000.

[HKST15] Juha Heinonen, Pekka Koskela, Nageswari Shanmugalingam, and Jeremy T. Tyson. Soboler spaces on metric measure spaces, volume 27 of New Mathematical Monographs. Cambridge University Press, Cambridge, 2015. An approach based on upper gradients.

[Ka199] Agnieszka Kałamajska. On compactness of embedding for Sobolev spaces defined on metric spaces. Ann. Acad. Sci. Fenn. Math., 24(1):123-132, 1999.

[Kei04] Stephen Keith. A differentiable structure for metric measure spaces. Adv. Math., 183(2):271315, 2004.

[KM16] Bruce Kleiner and John M. Mackay. Differentiable structures on metric measure spaces: a primer. Ann. Sc. Norm. Super. Pisa Cl. Sci. (5), 16(1):41-64, 2016.

[MPSC20] A. Maione, A. Pinamonti, and F. Serra Cassano. Г-convergence for functionals depending on vector fields. I. Integral representation and compactness. J. Math. Pures Appl. (9), 139:109-142, 2020.

[MV20] A. Maione and E. Vecchi. Integral representation of local left-invariant functionals in Carnot groups. Anal. Geom. Metr. Spaces, 8(1):1-14, 2020.

[SB74] M.-F. Sainte-Beuve. On the extension of von Neumann-Aumann's theorem. J. Functional Analysis, 17:112-129, 1974.

[Sha00] Nageswari Shanmugalingam. Newtonian spaces: an extension of Sobolev spaces to metric measure spaces. Rev. Mat. Iberoamericana, 16(2):243-279, 2000.

[Zhi02] V. V. Zhikov. Averaging of problems in the theory of elasticity on singular structures. Izv. Ross. Akad. Nauk Ser. Mat., 66(2):81-148, 2002. 